\title{
Novel neutralization mechanism of a human antibody with pan-coronavirus reactivity
}

Xiaoyu Sun

Shanghai Institute of Biochemistry and Cell Biology

Chunyan Yi

Yuanfei Zhu

Longfei Ding

Shuai Xia

Chenjian Gu

Xiao Lu

Shuangfeng Chen

Zhuo Yang

Yaguang Zhang

Liyan Ma

Wangpeng Gu

Mu Liu

Gaowei Hu

Shujuan Du

Renhong Yan

Weihui Fu

Songhua Yuan

Chenli Qiu

Chen Zhao

Xiaoyan Zhang

Yonghui He

Aidong Qu

Xu Zhou

Xiuling Li

Qiang Deng

Qiang Zhou

Hongzhou Lu

Zhiyang Ling ( $\sim$ lingzhiyang@sibs.ac.cn )

Shanghai Institute of Biochemistry and Cell Biology

Lu Lu ( $\square$ lul@fudan.edu.cn )

Fudan University

Jianqing Xu ( $\sim$ xujianqing@fudan.edu.cn ) 
Shanghai Public Health Clinical Center

Youhua Xie ( $\nabla$ yhxie@fudan.edu.cn )

Fudan University

Bing Sun ( $\sim$ bsun@sibs.ac.cn )

Shanghai Institute of Biochemistry and Cell Biology

\section{Research Article}

Keywords: Human antibody, pan-coronavirus reactivity, conserved epitope, fusion peptide and S2' cleavage site

Posted Date: October 1st, 2021

DOl: https://doi.org/10.21203/rs.3.rs-952553/v1

License: (c) (1) This work is licensed under a Creative Commons Attribution 4.0 International License. Read Full License 


\section{Abstract}

The recurrent outbreak of coronaviruses and variants underscores the need for broadly reactive antivirals and vaccines. Here, a novel broad-spectrum human antibody named 76E1 was isolated from a COVID-19 convalescent patient and showed broad neutralization activity against multiple $\alpha$ - and $\beta$-coronaviruses, including the SARS-CoV-2 variants and also exhibited the binding breath to peptides containing the epitope from $Y$ - and $\delta$ - coronaviruses. 76E1 cross-protects mice from SARS-CoV-2 and HCoV-OC43 infection in both prophylactic and treatment models. The epitope including the fusion peptide and S2' cleavage site recognized by $76 \mathrm{E} 1$ was significantly conserved among $\alpha-, \beta-, \gamma$ - and $\delta$ - coronaviruses. We uncovered a novel mechanism of antibody neutralization that the epitope of $76 \mathrm{E} 1$ was proportionally less exposed in the prefusion trimeric structure of spike protein but could be unmasked by binding to the receptor ACE2. Once the epitope exposed, 76E1 inhibited S2' cleavage, thus blocked the membrane fusion process. Our data demonstrate a key epitope targeted by broadly-neutralizing antibodies and will guide next-generation epitope-based pan-coronavirus vaccine design.

\section{Main}

Based on antigenic and genetic criteria, coronaviruses are organised into four genera: $\alpha-, \beta-, \gamma$ - and $\delta$ coronaviruses $^{1}$. In the first two decades of the 21 st century, there have been three major public health threats caused by highly pathogenic coronaviruses: severe acute respiratory syndrome coronavirus (SARS-CoV) in 2002-20032,3, Middle East respiratory syndrome coronavirus (MERS-CoV) in $2012^{4}$ and SARS-CoV-2, first identified in 20195,6. The COVID-19 pandemic caused by SARS-CoV-2 has had a profound impact on the world economy and global health. In addition, several common epidemic human coronaviruses including the $\beta$-coronaviruses $\mathrm{HCoV}-\mathrm{OC} 43, \mathrm{HCOV}-\mathrm{HKU} 1$ and the distantly related $\mathrm{a}-$ coronaviruses HCoV-229E, HCoV-NL63 circulate annually and cause mild or moderate upper respiratory diseases ${ }^{7,8}$. The recurrent spillover events of coronaviruses into humans highlight the need to develop broad coronavirus therapeutics and preventions.

One current research focus is the mutation of the SARS-CoV-2 genome during transmission ${ }^{9}$. A series of mutants have been identified within the spike (S) protein, which may weaken the protective activity of vaccines or antibodies that were designed based on the original sequence of the SARS-CoV-2 genome $^{10,11}$. The identification of resistant variants (e.g., the B.1.351 lineage) suggests potential risks in the application of current vaccines and antibodies; a new round of development may be needed once the resistant strain becomes dominant ${ }^{12,13}$.

Most neutralizing antibodies against SARS-CoV-2, SARS-CoV and MERS-CoV target the receptor binding domain (RBD) of $\mathrm{S} 1$, thus blocking the attachment of the viruses to target cells. However, RBD antibodies are usually subgenus or strain specific, and pose a selective pressure causing the rapid emergence of new variants ${ }^{14-17}$. Nevertheless, only a few antibodies targeting the RBD of SARS-CoV-2 have shown a narrow cross-reactivity which is limited within $\beta$-coronavirus genus ${ }^{18-20}$. Broadly neutralizing antibodies 
targeting conserved functional domains of viral surface proteins have been discovered to protect against global circulating viruses, such as IAV, HCV and HIV ${ }^{21-27}$. In addition, the $\mathrm{S} 2$ subunit is more conserved than that of $\mathrm{S} 1$ among diverse coronaviruses. Antibodies binding to the conserved S2 subunit have shown cross-reactivity to $\beta$-coronaviruses, but not a-coronaviruses as reported for human and mouse derived antibodies by targeting the conserved stem helix region $20,28-31$. RBD targeting-bnAbs ADG-2, H014 and S2X259 neutralize viruses by block virus receptor binding while the S2 bnAbs B6 and S2P6 were reported to disrupt the stem helix bundle, prevent S2 subunit refolding from the pre- to the postfusion state and thus block virus entry though inhibiting membrane fusion ${ }^{18,19,29,31,32}$. Here, we report a human antibody 76E1 with cross-neutralization to the representative strains from $\alpha$ - and $\beta$ coronavirus and even cross-binding activity to the peptides containing the antibody epitope from $Y$ - and $\delta$ coronavirus genera. This antibody showed cross-protection in mice against SARS-CoV-2 and phylogenetically distant $\mathrm{HCOV}-\mathrm{OC} 43$. The epitope including the fusion peptide and S2' cleavage site recognized by $76 \mathrm{E} 1$ was significantly conserved among $\alpha-, \beta-, \gamma$ - and $\delta$-coronaviruses. The recognition pattern is also interesting as the epitope is less exposed in the prefusion spike trimeric structure but could be unmasked by binding to the receptor ACE2 and followed by 76E1 binding and S2' cleavage inhibition. The identification of the conserved epitopes may guide the design of pan-coronavirus vaccine against the both current and future emerging SARS-CoV-2 variants and other coronavirus genera.

\section{Results}

\section{Isolation of S2-targeting human antibodies}

We initially measured the antigen binding potencies of the plasma from five COVID-19 convalescent patients as compared with that of healthy people. The $\mathrm{S} 2$ regions are conserved among multiple human coronavirus (Extended Data Fig. 1a). Antibodies that were cross-reactive with the S2 regions of SARSCoV-2, SARS-CoV and MERS-CoV were detected, especially in sample 76 (Extended Data Fig.1b-d). The tested plasma also reacted well to the spike proteins of HCoV-NL63, HCoV-OC43, HCoV-229E and HCoVHKU-1, with higher antibody titres than the healthy donor plasma (Extended Data Fig. 1e), indicating the potential existence of broad neutralizing antibodies, mostly targeting the $S 2$ region in COVID-19 convalescent patients.

We then conducted single-cell PCR experiments to isolate human monoclonal antibodies from the memory B cells of the COVID-19 convalescent patients by using the SARS-CoV-2 extracellular domain of spike protein (S-ECD) as the bait. We identified 25 specific antibodies, among which 9 reacted with the SARS-CoV-2 S2 (Supplementary Table 1-2). Three antibodies (555D6, 125C1, 76E1) showed cross-reactivity with both SARS-CoV and MERS-CoV S2/S-ECD. Most of the S2 antibodies could not neutralize SARS-CoV-2 pseudovirus, except for 76E1 and 555E6, which had IC50s of $0.418 \mu \mathrm{g} / \mathrm{ml}$ and $9.79 \mu \mathrm{g} / \mathrm{ml}$, respectively (Supplementary Table 1). 76E1 was derived from donor 76 and engages IGHV3$43 * 02$ and IGLV2-8*01 as the germline genes (Supplementary Table 3).

\section{Potency of 76E1 against SARS-CoV-2 and its variants}


76E1 was selected for further in-depth characterization and found to react well with the spike proteins of SARS-CoV-2 variants of concern including B.1.1.7 (WHO label: Alpha), B.1.351 (WHO label: Beta), P.1 (WHO label: Gamma) and B.1.617.1 (WHO label: Kappa) (Fig. 1a,c and Supplementary Fig. 1a-e) and we subsequently observed that 76E1 could still neutralize all the pseudotyped SARS-CoV-2 variants tested (Fig. 1b-c). Nevertheless, many RBD-targeting antibodies has partially or completely lost their neutralizing efficacy against some of the variants ${ }^{33}$. Consistent with the pseudovirus assay, 76E1 has shown similar potency in Vero-E6, HeLa-hACE2 and Calu-3 cells against authentic SARS-CoV-2 with IC50s ranging from 0.373 to $0.727 \mu \mathrm{g} / \mathrm{ml}$ (Fig. $1 \mathrm{~d})$.

To evaluate the prophylactic efficacy of 76E1 in vivo, human ACE2 transgenic mice were intraperitoneally (i.p.) administered $15 \mathrm{mg} / \mathrm{kg} \mathrm{mAb}$ or PBS $24 \mathrm{~h}$ before challenge with a dose of $10^{4}$ PFU SARS-CoV-2 via intranasal inoculation. All mice were monitored for body weight daily and euthanized at 3 days post infection (d.p.i) to allow for measurement of lung virus titres (Fig. 1e). Mice pretreated with $15 \mathrm{mg} / \mathrm{kg}$ $76 \mathrm{E} 1$ showed minor weight loss and apparent reduced lung viral titres with $10^{4}$ times compared to those of control mice (Fig. 1f-g). Prophylactically, 50 and $150 \mathrm{mg} / \mathrm{kg} 76 \mathrm{E} 1$ exhibited similar protective efficacy in weight loss and lung virus titre (Extended Data Fig. 2a-d). For posttreatment experiment, the mice were treated with $25 \mathrm{mg} / \mathrm{kg}$ of mAbs 8 hours after intranasal challenge with a 104-PFU dose of SARS-CoV-2 (Fig. 1e). Mice posttreated with both 76E1 also displayed minor weight loss and $10^{3}$ times lower lung virus titre than that of control mice (Fig. 1h-i). We conclude that 76E1 treatment can reduce virus burden in mice infected with SARS-CoV-2.

\section{Broad activity of 76E1 against multiple human coronaviruses}

There are seven coronaviruses have ever historically spilled over into humans and caused sever or mild diseases. We further assessed the broad reactivity of 76E1 to the spike proteins of the seven human coronaviruses consisting of two a-coronaviruses (HCoV-229E and HCoV-NL63) and five $\beta$-coronaviruses (SARS-CoV-2, SARS-CoV, MERS-CoV, HCoV-OC43 and HCoV-HKU1) (Fig. 2a). Using surface plasmon resonance (SPR) and ELISA, we found 76E1 bound to all the spike proteins tested with high binding activity (Fig. 2b and Supplementary Fig. 2a-b). Moreover, 76E1 neutralized the pseudotyped or authentic viruses with IC50s ranging from 0.373 to $4.821 \mu \mathrm{g} / \mathrm{ml}$ (Fig. 2c-h). To determine the cross-protective efficacy of 76E1 on HCoV-OC43 infection mouse model, the newborn mice were prophylactically treated with a dose of 15 or $50 \mathrm{mg} / \mathrm{kg}$ of $76 \mathrm{E} 1,50 \mathrm{mg} / \mathrm{kg}$ of isotype control antibody or with PBS, respectively, before challenge with a lethal dose of HCoV-OC43 and monitored daily for weight loss and survival rate for 14 days. Another groups of mice were euthanized at the 5 th day to determine the viral titres in brains. $50 \mathrm{mg} / \mathrm{kg} 76 \mathrm{E} 1$ was completely sufficient to protect mice from weight loss and death, while the body weight of mice pretreated with $15 \mathrm{mg} / \mathrm{kg}$ slightly decreased since day 7 and recovered soon in day 9 , resulting in $66.7 \%$ protection (Fig. $2 \mathrm{i}-\mathrm{j}$ ). For the posttreatment experiment, $50 \mathrm{mg} / \mathrm{kg} 76 \mathrm{E} 1$ resulted in $50 \%$ survival protection in mice (Fig. 2l-m). Moreover, 76E1 treatment also resulted in a significant reduction in brain viral loads at the 5th day, compared with the isotype control and PBS groups (Fig. 2k and 2n).

\section{E1 targets a conserved epitope within S2' cleavage site and the fusion peptide}


We initially found that 76E1 reacted well with the denatured S-ECD protein (Extended Data Fig. 3a). To identify the functionally defined epitopes recognized by $76 \mathrm{E} 1$, we performed amino acid sequence alignment of the spike proteins from 2 a-coronaviruses and $5 \beta$-coronaviruses and identified 7 conserved S2 sequences with peptide ${ }_{809-833}$ (SAS-CoV-2 numbering) showing reactivity to 76E1 (Extended Data Fig. 3b-c, 4 and Supplementary Table 4). Further experiments shorten the epitope into peptide ${ }_{809-823}$ (Extended Data Fig. 3d), containing S2' cleavage site (R815) and the fusion peptide (Fig. 3a), which could not compete 76E1, further conforming the epitope in peptide ${ }_{809-823}$ of 76E1 (Fig. 3b). To map the critical functional epitope residues for 76E1 binding, we performed ELISA-based epitope alanine scanning mutagenesis on the peptide ${ }_{809-823}$. Three mutants, R815A, E819A, and F823A, showed complete loss of reactivity to $76 \mathrm{E} 1$, validating the significance of the interaction between $76 \mathrm{E} 1$ and these residues. Additionally, D820A and L822A partially disrupted the binding to 76E1 (Fig. 3c). These sites are extremely conserved among all the sequences aligned from four coronavirus genera, including $\alpha-, \beta-, \gamma$ - and $\delta$ coronavirus (Fig. 3a). Of note, R815 is the S2' cleavage site, which is critical for the cleavage event that initiates fusion activation ${ }^{34,35}$.

However, 76E1 could not efficiently recognize the trimeric prefusion spike protein of SARS-CoV-2 even at $1000 \mathrm{nM}$, while the RBD antibody 76F6 at $50 \mathrm{nM}$ could achieve high binding response (Fig. 3d, e). Referring to the structure of the prefusion spike ${ }^{35}$, the 76E1 epitope was partially buried in the prefusion trimeric structure (Fig. 3f). A loop upstream of the S2' cleavage site was stabilized by three prolines (P807, P809 and P812) and seemed to partially shield S2' cleavage site and the fusion peptide. Some of the side chains of the critical epitope residues adopted orientations towards the inside of the spike stem region, and were thus difficult to access directly (Fig. 3f). These results may explain why 76E1 could not recognize the prefusion state of the SARS-CoV-2 spike protein. We also compared the functionally defined epitopes of 76E1 within other $\alpha$ - and $\beta$-coronaviruses and found that the tertiary structure of the region was quite conserved, providing the structural basis for the broad neutralization ability of 76E1 (Extended Data Fig. 5a-e).

As expected, 76E1 bound well to the peptides ${ }_{809-823}$ from a- and $\beta$-coronaviruses (Fig. 3g-h), it also showed high reactivity to peptides ${ }_{809-823}$ from representative strains of $\gamma$ - and $\delta$-coronaviruses (Fig. 3i-j), indicating a unprecedent breadth of this antibody.

\section{E1 inhibits membrane fusion by inhibiting S2' cleavage.}

Considering that 76E1 targets S2' cleavage site and the fusion peptide, we speculated that 76E1 might neutralize coronaviruses by blocking the process of membrane fusion. To address this hypothesis, a membrane fusion assay involving spike-expressing HeLa cells and ACE2expressing HeLa cells was performed. As expected, 76E1 strongly prevented membrane fusion induced by the $S$ proteins of SARS-CoV, SARS-CoV-2 and MERS-CoV, while the isotype control antibody showed no inhibitory effect (Fig. 4a). In accordance with the reported publications ${ }^{36-38}$, the 
degree of fusion induced by SARS-CoV-2 is higher than that of MERS-CoV and SARS-CoV, reflecting the strong transmissibility of SARS-CoV-2.

During viral replication, the spike protein is synthesized initially as a precursor protein and then processed at the S1/S2 cleavage site by host cell proteases, which cut the S protein into S1 and S2 regions that still combine by noncovalent binding. Cleavage at the S2' site triggers significant conformational rearrangements, leading to the exposure of the fusion peptide, thus promoting the fusion of the host cell and viral membranes and consequently resulting in virus entry ${ }^{8,39-41}$. Since the S2' cleavage site (R815) is one of the key epitope residues of 76E1, we hypothesized that inhibiting S2' cleavage is a possible mechanism by which 76E1 blocks membrane fusion. The C-terminal-His-tagged soluble S-ECDs of SARSCoV-2, SARS-CoV and MERS-CoV were incubated with 76E1 or control antibody before treatment with trypsin. As expected, 76E1 could not efficiently inhibit S1/S2 cleavage but dramatically impaired S2' cleavage of soluble S-ECDs in a time-dependent manner, resulting in fewer S2' fragments than the control antibody (Fig. 4b-d). These results suggested that the neutralizing activity of 76E1 was possibly mediated by inhibiting $\mathbf{S 2}$ ' cleavage.

\section{ACE2 promotes the exposure of the 76E1 epitope in SARS-CoV-2}

Fusion activation is considered to involve the exposure and cleavage of the S2' site, which contributes to the release of the fusion peptide. Other groups proposed that receptor binding would promote the exposure of the S2' cleavage site and fusion peptide ${ }^{34,37,42}$, which is also the epitope of 76E1. Considering 76E1 could not effectively bind the prefusion spike trimer, we proposed that the recognition of spike to ACE2 may promote the exposure of the 76E1 epitope in SARS-CoV-2. To address this question, A549 cells expressing full-length SARS-CoV-2 S were treated with recombinant human ACE2 (hACE2) protein before trypsin cleavage. Interestingly, recombinant hACE2 protein significantly increased S2' cleavage by trypsin, resulting in the release of more S2' fragments than observed in the control (Fig. 5a). Notably, 76E1 competed with trypsin and inhibited hACE2-induced S2' cleavage (Fig. 5b). We further performed flow cytometry to determine whether hACE2 could enhance the binding of 76E1 to cellsurface-expressed native spike proteins. A substantially higher frequency of 76E1 binding (1.5-fold) and mean fluorescence intensity (4-fold) were observed for hACE2-treated cells than for untreated cells. The number of RBD antibody-binding cells decreased upon treatment with hACE2, as hACE2 competes with this antibody for the epitope on the spike (Fig. 5c-d). In conclusion, the 76E1 epitope in the trimer interface was less frequently or proportionally less exposed but could be unmasked by the hACE2dependent receptor binding process, thus favouring the neutralization of viruses.

We further determined whether 76E1 is synergistic with hACE2 in neutralizing authentic SARS-CoV-2 using the CompuSyn program which has been described previously ${ }^{43,44}$. Dose-dependent neutralizing activity of 76E1, hACE2, or their combination was then evaluated by serial threefold dilutions in concentrations from 81 times the IC50 (Fig. 5e). The combination index (Cl) was analysed to evaluate the degree of cooperation among the two drugs using the CompuSyn program. The $\mathrm{Cl}$ values of the mixture at ED50, ED75 and ED90 were presented as $0.623,0.67$ and 0.722 , respectively, which are less than 1 , a 
hallmark of synergism (Fig. $5 f$ and Extended Data Table 1). The dose reduction index (DRI) was calculated by comparing the dose required to reach percent neutralization when 76E1 or hACE2 was treated alone and in combination and the data showed that combined 76E1 and hACE2 was 2.474 3.687-fold reduction at IC50, IC75 or IC90, further demonstrating the synergy of 76E1 and hACE2 (Extended Data Table 1).

\section{Discussion}

The $\mathrm{S} 2$ region of coronavirus spike protein is more conserved than the $\mathrm{S} 1$ region and thus is a promising target for broad coronavirus antibodies and vaccines. Several reported S2 antibodies, such as S2P6, B6, 28D9 and CC40.8 have shown cross-reactivity to $\beta$-coronaviruses, but not other coronavirus genera, by targeting the conserved stem helix region and block the fusion machinery $20,28-30$. Although Chek Meng Poh et al has described a linear peptide in the fusion peptide that is recognized by convalescent COVID19 plasmas, they have not isolated monoclonal antibodies targeting this peptide and no data were revealed the working mechanisms related to antibodies targeting fusion peptide ${ }^{45}$. However, in our work, $76 \mathrm{E} 1$ shows an unprecedented broad reactivity against pan-coronavirus genera, including $\beta$-coronavirus as well as $\alpha$-coronavirus, even several tested $\gamma$ - and $\delta$ - coronaviruses. The high conservation of the epitope recognized by 76E1 determined the broad binding potency of this antibody. The IC50 of 76E1 against authentic SARS-CoV-2 was higher than some of the most potent RBD antibodies. Indeed, the reported S2 targeting antibodies also showed moderate neutralization activity with IC50s in $\mu \mathrm{g}$ range ${ }^{28-}$

30 . This is also consistent with reports for influenza virus, in which the neutralizing potency of antibodies targeting the immunodominant HA (haemagglutinin) head region is significantly higher than that of antibodies against the stem region ${ }^{21}$. Our in vivo data showed that 76E1 displayed protection in reducing the lung virus titre, indicating the potential for clinical application. The broad spectrum defines 76E1 as an attractive reagent to combat the challenge of antibody escape mutations and cope with a possible novel coronavirus outbreak in the not-too-distant future.

The functionally defined epitope of 76E1 includes several amino acids that are conserved among $\alpha-, \beta-, \gamma^{-}$ and $\delta$-coronaviruses, including R815, E819, D820 and L823 (SARS-CoV-2 numbering). It must be emphasized that the key residue R815 is the "fusion activation" proteolytic site (S2') of SARS-CoV-2. Thus, the possible neutralizing mechanism of 76E1 is its inhibition of the cleavage of the S2' site. Our work indicates that the $S 2$ ' cleavage site is a promising target for designing inhibitors and antibody drugs.

The SARS-CoV-2 spike protein is produced in a compact, closed form in which the helices in the S2 membrane fusion component are capped by the RBD of neighbouring monomers ${ }^{34}$. This is consistent with the result that 76E1 barely recognizes the stable trimeric SARS-CoV-2 S prefusion protein. The moderate neutralization potency of S2 antibodies such as S2P6 and 76E1 may possibly as a result of limited epitope exposure and different neutralizing mechanism. ACE2 promoted the exposure of S2' cleavage site and the fusion peptide, perhaps by structural rearrangement of the spike protein, thus 
removing the obstacles shielding this epitope and providing accessibility for the protease and 76E1 ${ }^{34,37}$. We also provide a potential combination of 76E1 and hACE2 to prevent SARS-CoV-2 infection.

Epitope-based vaccine design has shed light for respiratory syncytial virus, influenza virus and other viruses $^{46,47}$. We further found that the antibodies recognizing the peptide ${ }_{809-823}$ could be widely induced in the COVID-19 convalescent patients (Extended Data Fig. 6a-b). Our data demonstrate a promising epitope for next-generation epitope-based broad coronavirus vaccine design.

\section{Materials And Methods}

\section{Cells and viruses}

HeLa, RD, LLC-MK2, HuH-7 and HEK 293T cells were all obtained from the American Type Culture collection (Manassas, VA, USA) and cultured in Dulbecco's modified Eagle's medium (DMEM, Gibco) with $10 \%$ foetal bovine serum (FBS). CHO cells were purchased from Thermo Fisher Scientific and cultured in Expi $^{\text {TM }} \mathrm{CHO}$ expression medium (Thermo Fisher Scientific, cat. A2910001). HEK 293T cells stably expressing human ACE2 (293T-hACE2) were generated and cultured in DMEM with 10\% FBS. A549 cells stably expressing the spike gene of SARS-CoV-2 (GenBank: MT121215.1) were generated and cultured in DMEM with $10 \%$ FBS. The SARS-CoV-2 clinical isolate nCoV-SH01 was amplified in VeroE6 cells and was used for the authentic virus neutralization assay in vitro and protective efficacy assay in vivo.

\section{Recombinant proteins and antibodies}

To generate recombinant human ACE2 protein, a DNA fragment encoding the extracellular domain of human ACE2 (residues S19 to S740) was cloned into a refined pGT128 vector containing an antibody signal peptide in the N-terminus and human IgG1 Fc in the C-terminus. A flexible "GSGGGG" linker was inserted between ACE2 and human IgG1 Fc. The recombinant hACE2 protein was expressed in $\mathrm{CHO}$ cells and purified with Protein A (MabSelect Prism A, GE Healthcare) and size exclusion chromatography using a Superdex 200 10/300 column (GE Healthcare).

To generate the prefusion trimeric spike protein, the extracellular domain of the S protein (1-1208 amino acids) was cloned into the PCAG vector with a "GSAS" substitution at residues 682 to 685 , two proline substitutions at residues 986 and 987, and a T4 fibritin trimerization motif in the C-terminus.

The following spike proteins were expressed either from baculovirus or mammalian expression systems and utilized for binding activity assays. The S-ECD is the wild-type extracellular domain of the spike protein lacking the transmembrane region (TM) and C-terminal T4 fibritin trimerization motif and without any mutations in the sequence: SARS-CoV-2 S-ECD-His (Sino Biological, Cat: 40589-V08B1), SARS-CoV SECD-His (Sino Biological, Cat: 40634-V08B ), MERS-CoV-2 S-ECD-His (Sino Biological, Cat: 40069-V08B), HCoV-NL63 S-ECD-His (Sino Biological, Cat: 40604-V08B), HCoV-229E S-ECD-His (Sino Biological, Cat: 40605-V08B), HCoV-OC43 S-ECD-His (Sino Biological, Cat: 40607-V08B), HCoV-HKU1 S-ECD-His (Sino Biological, Cat: 40606-V08B), SARS-CoV-2 S1-His (Sino Biological, Cat: 40150-V08B1), SARS-CoV-2 S2- 
His (Sino Biological, Cat: 40590-V08B), SARS-CoV-2 RBD-SD1-His (Novoprotein, Cat: DRA42), SARS-CoV S1-His (Sino Biological, Cat: 40150-V08B1), SARS-CoV S2-His (Sino Biological, Cat: 40150-V08B3), SARSCoV RBD-His (Sino Biological, Cat: 40150-V08B2), MERS-CoV S1-His (Sino Biological, Cat: 40069-V08H), MERS-CoV S2-His (Sino Biological, Cat: 40070-V08B), and MERS-CoV RBD-His (Sino Biological, Cat: 40071-V08B1).

\section{Isolation, cloning, expression and purification of 76E1}

This study was approved by the Ethnic Review Board of Shanghai Public Health Clinical Center. Blood was collected from patients who had recovered from SARS-CoV-2 infection after they had signed the informed consent form. Fresh PBMCs were isolated from the collected blood by using the Ficoll-Paque gradient (GE Healthcare, cat. no.17144002). Percp-CY5.5-CD4;, Percp-CY5.5-CD14; Percp-CY5.5-CD8- and APC-Cy7-780-dead' and FITC-CD19+ ${ }^{+}$APC-IgG ${ }^{+}$, biotinylated SARS-CoV-2 spike protein ${ }^{+}$-streptavidin-SA BV $421^{+}$specific single memory B cells were isolated by a Sony MA900 flow separator and sorted into $96-$ well plates. The plasmids encoding the heavy chain and light chain of the antibodies were cotransfected into Expi ${ }^{\mathrm{TM}} \mathrm{CHO}$ cells, which were cultured and harvested according to the manufacturer's instructions (Thermo Fisher Scientific, cat. no. A29129). The antibodies used in the functional assays were all purified from the Expi ${ }^{\mathrm{TM}} \mathrm{CHO}$ cell supernatants using Protein $\mathrm{A}$ and followed by size exclusion chromatography (Superdex 200 10/300 column).

\section{ELISA}

To determine the binding properties of the antibodies and sera, spike proteins $(0.5 \mu \mathrm{g} / \mathrm{ml}$ in $100 \mu \mathrm{l} / \mathrm{well})$ were captured on 96 -well plates overnight and blocked with $2 \%$ bovine serum albumin in PBS-Tween 20 (PBST) for $2 \mathrm{~h}$. Antibodies or sera were serially diluted and incubated in the wells for $2 \mathrm{~h}$. The samples were washed three times, and an anti-human Fc-HRP antibody (Sigma-Aldrich) was used to determine the binding affinity, followed by incubation with tetramethylbenzidine (Beyotime), which was stopped by adding $1 \mathrm{M} \mathrm{HCl}$. The absorbance at $450 \mathrm{~nm}$ was recorded by a plate reader (Bio-tek). For the receptor competition assay, hACE2 $(5 \mu \mathrm{g} / \mathrm{ml})$ was coated on the microplates. The isolated antibodies were serially diluted and incubated with biotinylated spike protein $(0.3 \mu \mathrm{g} / \mathrm{ml})$ for $1 \mathrm{~h}$ and then added to the wells, after washing and blocking. Streptavidin-conjugated HRP was used as the detection antibody. The following procedure was the same as mentioned above. To evaluate the reactivity of 76E1 to denatured or WT spike protein, ELISA was performed as mentioned above. Spike protein was denatured with $0.1 \%$ SDS, 50 $\mathrm{mM}$ DTT in a metal block at $100^{\circ} \mathrm{C}$ for $5 \mathrm{~min}$.

\section{Biolayer interferometry (BLI) analysis of spike proteins and antibodies}

Biolayer interferometry was performed using an Octet Red instrument (ForteBio, Inc.). To test the binding affinity between 76E1 and S-ECD, 76E1 $(15 \mu \mathrm{g} / \mathrm{ml})$ was immobilized on an anti-human lgG-Fc-coated biosensor (AHC) surface for $300 \mathrm{sec}$. The baseline interference phase was then read for $180 \mathrm{sec}$ in kinetics buffer (KB: $1 \times$ PBS), followed by subsequent association phase immersion of the sensors into wells containing two-fold serial dilutions of S-ECD protein (from $200 \mathrm{nM}$ to $6.25 \mathrm{nM}$ ) in KB for $600 \mathrm{sec}$. 
Then, the sensors were immersed in $\mathrm{KB}$ for dissociation for up to $600 \mathrm{sec}$. The mean $\mathrm{K}_{\mathrm{on}}, \mathrm{K}_{\text {off }}$ and apparent KD values of 76E1 binding affinity with different human coronavirus spike proteins were calculated using the global fit to a 1:1 Langmuir binding model with an $R^{2}$ value of $\geq 0.95$. To determine the affinity of $76 \mathrm{E} 1$ to the prefusion trimeric spike protein, biotinylated trimeric spike protein $(15 \mu \mathrm{g} / \mathrm{ml})$ was immobilized on a streptavidin biosensor for $300 \mathrm{sec}$. The following phases were as described above except for the association phase, in which the sensors were immersed into wells containing serially diluted 76E1 (1000-500-250-125-62.5 nM) or control antibody 76F6 (50-25-12.5-6.25-1 nM). To determine

whether the spike protein can compete the binding to 76E1 in the presence of peptide ${ }_{809-823}, 76 \mathrm{E} 1$ (15 $\mu \mathrm{g} / \mathrm{ml}$ ) was immobilized on an AHC sensor for $300 \mathrm{sec}$. The baseline interference phase was then read for $180 \mathrm{sec}$ in $\mathrm{KB}$, followed by subsequent association phase immersion of the sensors into wells containing peptide ${ }_{809-823}$ for $240 \mathrm{sec}$. Then, the sensors were immersed in KB containing spike protein $(100 \mathrm{nM})$ for $360 \mathrm{sec}$.

\section{Pseudovirus neutralization assay}

The percent neutralization activity of 76E1 was first determined against SARS-CoV, SARS-CoV-2, MERS$\mathrm{CoV}$ and HCoV-229E pseudoviruses using a method as previously described ${ }^{48}$. Briefly, plasmids encoding the full-length $S$ gene and pNL4-3.luc.RE were cotransfected into HEK 293T cells in $10 \mathrm{~cm}$ dishes. The medium was replaced after $6 \mathrm{~h}$, and virus supernatants were collected $48 \mathrm{~h}$ after transfection. HEK 293ThACE2 cells were plated in 96-well plates and grown overnight. The virus was then diluted in DMEM with $10 \%$ FBS mixed with an equal volume of serially diluted antibodies and incubated at $37^{\circ} \mathrm{C}$. One hour later, the mixtures were transferred to HEK 293T-hACE2 cells in 96-well plates. The cells were incubated at $37^{\circ} \mathrm{C}$ for $48 \mathrm{~h}$, followed by cell lysis and luciferase activity assays (Promega). The percent neutralization was calculated by comparing the luciferase value of the antibody-treated cells to that of the untreated control.

\section{Authentic virus neutralization assay}

The authentic SARS-CoV-2 experiments were performed in the BSL-3 laboratory of Fudan University under the guidelines of Fudan University. Vero-E6, HeLa-hACE2 and Calu-3 cells were plated into 96-well plates and grown overnight. One hundred TCID50 $(50 \mu \mathrm{L}$ ) of live SARS-CoV-2 virus (nCoV-SH01) was mixed with $50 \mu \mathrm{L}$ of threefold serially diluted $76 \mathrm{E} 1$ and incubated at $37^{\circ} \mathrm{C}$ for $1 \mathrm{~h}$. The supernatants were discarded, and the antibody-virus mixture was mixed with the target cells. After incubation at $37^{\circ} \mathrm{C}$ for $1 \mathrm{~h}$, the DMEM containing $1 \%$ methylcellulose and $2 \%$ FBS was added. The cells were incubated at $37^{\circ} \mathrm{C}$ for $24 \mathrm{~h}$, fixed with $1 \%$ formaldehyde for 1 hour, followed by three times of washing with PBS, and permeabilized in $0.2 \%$ Triton-X in PBS with gentle shaking for 10 minutes at RT. The wells were then blocked with $2 \%$ BSA for 2 hours at RT. The cells were first incubated with a primary antibody recognizing SARS-CoV-2 NP protein, followed by a secondary antibody incubation with AlexaFluor 488-conjugated goat anti-rabbit antibody. The data were collected by a high throughput imaging instrument Cytation/BioSpa8/Multiflo FX (CBM). The concentration of antibody required for a $50 \%$ decrease in foci number (IC50) was calculated using a nonlinear curve fitting algorithm in GraphPad Prism 7. 
The synergistic effect of 76E1 with hACE2 in neutralizing authentic SARS-CoV-2 in Vero-E6 cells was determined using a method that has been described previously ${ }^{43,44}$. 76E1, hACE2 and their combination were threefold serially diluted from 81 times the IC50, and incubated with SARS-CoV-2 virus (100 TCID50) at $37^{\circ} \mathrm{C}$ for $1 \mathrm{~h} .76 \mathrm{E} 1$ and $\mathrm{hACE} 2$ in the combination was mixed at a constant ratio, which represented the dose at IC50. The neutralization was performed as mentioned above. The combination index (CI) and dose reduction index (DRI) at ED50, ED75 and ED90 (effective dose at IC50, IC75 and IC90) were analysed to evaluate the degree of cooperation among the two drugs using the CompuSyn program.

The inhibitory activity of antibodies against HCoV-OC43 (ATCC, VR-1558) infection in RD cells was assessed. Briefly, 100 TCID50 of HCoV-OC43 was mixed with a test antibody at indicated concentration and incubated at $37^{\circ} \mathrm{C}$ for $30 \mathrm{~min}$. The mixture was added to RD cells. After culture for additional 2-3 days, Cell Counting Kit-8 (CCK8, Dojindo, Kumamoto, Kyushu, Japan) assay was used to determine cytopathic effect. The inhibitory activity of the tested antibodies against HCoV-229E (ATCC, VR-740) infection in Huh-7 cells and HCoV-NL63 (Amsterdam strain) infection in LLC-MK2 cells was evaluated in a similar way, as described above. IC50 was calculated using GraphPad Prism 7.

\section{Flow cytometry}

To assess whether hACE2 (recombinant human ACE2-human IgG1 Fc) promoted the binding activity of $76 \mathrm{E} 1$ to the spike protein, A549 cells stably expressing the spike protein of SARS-CoV-2 were detached with TrypLE ${ }^{\mathrm{TM}}$ Express (Gibco) and incubated with $20 \mu \mathrm{g} / \mathrm{ml}$ hACE2 on ice for $30 \mathrm{~min}$. After washing and staining with FVS780-APC-CY7, the cells were incubated with $15 \mu \mathrm{g} / \mathrm{ml}$ biotinylated 76E1, 76F6 or isotype control antibody in FACS buffer on ice for $1 \mathrm{~h}$. Antibodies still bound to surface-expressed spike protein after washing were then stained with streptavidin-conjugated BV421 (BD Biosciences), followed by flow cytometry. The MFI was calculated.

\section{Protective efficacy in mice}

To assess the protective efficacy of 76E1 in SARS-CoV-2 infection model, transgenic mice expressing human ACE2-luciferase driven by the CAG promoter, named Tgtn (CAG-human ACE2-IRES-Luciferase), were purchased from Shanghai Model Organisms and used to test the protective efficacy of 76E1. Briefly, 10-week-old female mice were raised in the BSL-3 laboratory of Fudan University and received humane care in compliance with the guidelines of the Animal Research Ethics Board of Fudan University. For prophylactic experiments, the mice were intranasally infected with SARS-CoV-2 $\left(1 \times 10^{4}\right.$ PFU in Figure 1 e and $3.4 \times 10^{4}$ PFU in Extended Data Figure 2) $24 \mathrm{~h}$ after i.p. administration of 76E1 (15mg/kg in Figure 1e and 150 or $50 \mathrm{mg} / \mathrm{kg}$ in Extended Data Figure 2). For posttreatment experiments, the mice were treated with 76E1 after SARS-CoV-2 infection. The weight was monitored for 3 days postinfection, the mice were euthanized at $3 \mathrm{dpi}$, and the lungs were collected for virus titre examination. PBS inoculation was included as controls. Viral RNA was extracted from lung tissue with TRIzol reagent (Invitrogen) and reverse transcribed into cDNA (Toyobo). Real-time qPCR was performed using a SYBR Green kit (Toyobo). The SARS-CoV-2 N gene-specific primers were as follows: forward primer, 5'- 
GGGGAACTTCTCCTGCTAGAAT-3'; reverse primer, 5'-CAGACATTTTGCTCTCAAGCTG-3'. The lung samples for histological examination were stored in $4 \%$ neutral-buffered formalin for seven days, embedded in paraffin, sectioned and stained with haematoxylin and eosin, followed by light microscopy.

To determine the protective efficacy of 76E1 against HCoV-OC43, pregnant BALB/c mice (18 days) were separated into four groups after delivery of their offspring and received humane care in compliance with the guidelines of the Animal Research Ethics Board of Fudan University. For prophylactic experiments, 4 days' newborn BALB/c mice were pretreated with 15 or $50 \mathrm{mg} / \mathrm{kg}$ antibodies or PBS $24 \mathrm{~h}$ before challenge with OC43 with a lethal dose. For posttreatment experiments, the 7-day-old mice were treated with 50 $\mathrm{mg} / \mathrm{kg}$ antibodies or PBS $2 \mathrm{~h}$ after challenge with OC43. The body weight and survival rate were monitored for 14 days postinfection. Another groups of mice were euthanized at $5 \mathrm{dpi}$, and the brains were collected for virus titre examination by calculating CPE on RD cells and expressed as TCID50/g.

\section{Syncytium inhibition assay}

The experimental procedure was previously described with some modifications ${ }^{48}$. In brief, HeLa cells were transfected (when $~ 60-70 \%$ confluent in six-well plates) by Lipofectamine 2000 with plasmids encoding codon-optimized full-length SARS-CoV, SARS-CoV-2 or MERS-CoV S proteins. Another group of HeLa cells was transfected with plasmids encoding human ACE2. Forty-eight hours after transfection, the $S$ protein group cells were detached and incubated with different concentrations of 76E1 or control antibody at $37^{\circ} \mathrm{C}$ for $1 \mathrm{~h}$. Then, the ACE2 group cells were detached and mixed with S protein group cells at a 1:1 ratio in 48-well plates. After $12 \mathrm{~h}$ of coculture, fused cells were observed. After washing and fixing with $4 \%$ paraformaldehyde, the cells were dyed with crystal violet. Images were photographed with an Olympus IX73 confocal microscope using a 10X objective lens.

\section{Trypsin cleavage inhibition assay}

Spike-histidine (S-ECD-His) protein $(0.1 \mu \mathrm{g}$, Sino Biological) was incubated in the presence of $0.4 \mu \mathrm{g} 76 \mathrm{E} 1$ or the control antibody in PBS at room temperature for $1 \mathrm{~h}$. Trypsin digestion was conducted by adding TPCK-treated trypsin (Sigma) to spike protein at a ratio of 1:200 (for MERS-CoV) or 1:100 (for SARS-CoV and SARS-CoV-2) and stopped at different time points by boiling the sample in a $100^{\circ} \mathrm{C}$ water bath. Samples were run on a 7.5-12\% SDS-polyacrylamide electrophoresis gel under reducing conditions and blotted using HRP-conjugated His probe-horseradish peroxidase (Proteintech) and SuperSignal ${ }^{\mathrm{TM}}$ West Pico PLUS Chemiluminescent Substrate (Thermo Fisher Scientific). Exposed images are presented in Figure. $4 \mathrm{~B}$ to $4 \mathrm{D}$.

\section{hACE2 promotes exposure of the S2' cleavage site}

To determine whether hACE2 promotes exposure of the S2' cleavage site, A549 cells stably expressing the spike protein of SARS-CoV-2 were plated into 12-well plates and incubated with 20 $\mu \mathrm{g} / \mathrm{ml} \mathrm{hACE2}$ at $37^{\circ} \mathrm{C}$ for $30 \mathrm{~min}$. After washing, the cells were digested with $0.4,0.2$ or $0.1 \mu \mathrm{g} / \mathrm{ml} \mathrm{TPCK}$ treated trypsin at $37^{\circ} \mathrm{C}$ for $15 \mathrm{~min}$. Then, the cells were washed twice again, and the cleavage were 
stopped at different time points by boiling the sample in a $100^{\circ} \mathrm{C}$ water bath. Samples were run on a 7.5$12 \%$ SDS-polyacrylamide electrophoresis gel under reducing conditions and blotted using a rabbit antiSARS-CoV-2 Spike S2 antibody (Sino Biological, Cat: 40590-T62). Recombinant hACE2 was detected by a goat anti-human IgG1 Fc-HRP antibody, and actin was detected by a mouse anti-actin antibody.

\section{Statistical analyses}

Statistical significance was analysed as described in the figure legends, and values of $P<0.05$ were considered statistically significant. Statistical analyses were performed using GraphPad Prism.

\section{Declarations}

\section{Acknowledgements}

We thank the Core Facilities of Chemical Biology, Cell Biology and Molecular Biology of Shanghai Institute of Biochemistry and Cell Biology, Center for Excellence in Molecular Cell Science for their help. This work was supported by the Ministry of Science and Technology of China (2018YFA0507402), the National Natural Science Foundation of China (82041015, 81971921, 81761128007 and 82041036), the Strategic Priority Research Program of the Chinese Academy of Sciences (XDB19000000), China Postdoctoral Science Foundation (NO.2020T130120ZX), the Key International Partnership Program of the Chinese Academy of Sciences (153D31KYSB20180055), the National Major Science and Technology Projects of China (2018ZX10301208), and Shanghai Science and Technology Commission (18DZ2293000).

\section{Author contributions}

B.S., Y.H.X., J.Q.X., L.L. and Z.Y.L. initiated, designed and supervised the study. X.Y.S. and C.Y.Y. designed and performed the all the experiments. Y.F.Z., S.J.D., S.X. and G.W.H. performed all the animal studies. L.F.D. conducted human serum-related experiments. L.F.D. and C.L.Q. contributed to the isolation of spikespecific memory B cells. C.J.G. and M.L. contributed to the authentic virus neutralization experiments. W.H.F., S.H.Y., C.Z. and X.Y.Z. collected the blood sample. R.H.Y., X.L., S.X., Y.G.Z., L.Y.M. and W.P.G. provided materials and helped with some experiments during this study. A.D.Q., X.Z. and X.L.L. prepared the recombinant hACE2 protein. B.S., Z.Y.L., X.Y.S., C.Y.Y. and S.X. analysed the data. Z.Y. performed structural analysis. Q.Z., H.Z.L. and Q.D. provided valuable suggestions. X.Y.S., C.Y.Y., Z.Y.L., and B.S. wrote the paper.

\section{Competing interests}

B.S., X.Y.S., C.Y.Y., Z.Y.L. and Y.G.Z. are listed as inventors on pending patent applications for 76E1. The pending patent of 76E1 has been licenced. The other authors declare no competing interests.

\section{Data and materials availability}


The spike protein structures used for analysis were 6XR8 (SARS-CoV-2) ${ }^{35}, 5$ X58 (SARS-CoV) ${ }^{49}, 5$ W9I $(\mathrm{MERS}-\mathrm{CoV})^{49}$, 5SZS (HCoV-NL63) ${ }^{50}, 6 \mathrm{NZK}(\mathrm{HCoV}-\mathrm{OC} 43)^{51}$, and 6U7H (HCoV-229E) ${ }^{52}$, which were downloaded from the NCBI Protein Data Bank (PDB). The spike sequences were downloaded from the National Center for Biotechnology Information (NCBI) database. The sequences used in supplementary figure1A and 6 were: SARS-CoV-2 (GenBank: MT121215.1), SARS-CoV (GenBank: ABF65836.1), MERSCoV (GenBank: AKN11072.1), HCoV-229E (GenBank: NP_073551.1), HCoV-NL63 (GenBank: YP_003767.1), HCoV-OC43 (GenBank: YP_009555241.1), HCoV-HKU1 (GenBank: ABD75513.1). The authors declare that all other data supporting the findings of this study are available within the article and its supplementary files.

\section{References}

1. Malik, Y.A. Properties of Coronavirus and SARS-CoV-2. Malays J Pathol 42, 3-11 (2020).

2. Drosten, C., et al. Identification of a novel coronavirus in patients with severe acute respiratory syndrome. N Engl J Med 348, 1967-1976 (2003).

3. Ksiazek, T.G., et al. A novel coronavirus associated with severe acute respiratory syndrome. N Engl J Med 348, 1953-1966 (2003).

4. Zaki, A.M., van Boheemen, S., Bestebroer, T.M., Osterhaus, A.D. \& Fouchier, R.A. Isolation of a novel coronavirus from a man with pneumonia in Saudi Arabia. N Engl J Med 367, 1814-1820 (2012).

5. Layne, S.P., Hyman, J.M., Morens, D.M. \& Taubenberger, J.K. New coronavirus outbreak: Framing questions for pandemic prevention. Sci Transl Med 12(2020).

6. Zhou, P., et al. A pneumonia outbreak associated with a new coronavirus of probable bat origin. Nature 579, 270-273 (2020).

7. Cui, J., Li, F. \& Shi, Z.L. Origin and evolution of pathogenic coronaviruses. Nat Rev Microbio/ 17, 181-192 (2019).

8. Walls, A.C., et al. Structure, Function, and Antigenicity of the SARS-CoV-2 Spike Glycoprotein. Cell 183,1735 (2020).

9. Layne, S.P. \& Taubenberger, J.K. Increasing threats from SARS-CoV-2 variants: Time to establish global surveillance. Sci Transl Med 13(2021).

10. Grubaugh, N.D., Hodcroft, E.B., Fauver, J.R., Phelan, A.L. \& Cevik, M. Public health actions to control new SARS-CoV-2 variants. Cell (2021).

11. Fenwick, C., et al. A high-throughput cell- and virus-free assay shows reduced neutralization of SARS-CoV-2 variants by COVID-19 convalescent plasma. Sci Transl Med 13(2021). 
12. Liu, Y., et al. Neutralizing Activity of BNT162b2-Elicited Serum - Preliminary Report. N Engl J Med (2021).

13. Wu, K., et al. Serum Neutralizing Activity Elicited by mRNA-1273 Vaccine - Preliminary Report. $N$ Engl J Med (2021).

14. Cao, Y., et al. Potent Neutralizing Antibodies against SARS-CoV-2 Identified by High-Throughput Single-Cell Sequencing of Convalescent Patients' B Cells. Cell 182, $73-84$ e16 (2020).

15. Shi, R., et al. A human neutralizing antibody targets the receptor-binding site of SARS-CoV-2. Nature 584, 120-124 (2020).

16. Wu, Y., et al. A noncompeting pair of human neutralizing antibodies block COVID-19 virus binding to its receptor ACE2. Science 368, 1274-1278 (2020).

17. Barnes, C.O., et al. Structures of Human Antibodies Bound to SARS-CoV-2 Spike Reveal Common Epitopes and Recurrent Features of Antibodies. Cell 182, 828-842 e816 (2020).

18. Rappazzo, C.G., et al. Broad and potent activity against SARS-like viruses by an engineered human monoclonal antibody. Science 371, 823-829 (2021).

19. Lv, Z., et al. Structural basis for neutralization of SARS-CoV-2 and SARS-CoV by a potent therapeutic antibody. Science 369, 1505-1509 (2020).

20. Shiakolas, A.R., et al. Cross-reactive coronavirus antibodies with diverse epitope specificities and Fc effector functions. Cell Rep Med 2, 100313 (2021).

21. Kallewaard, N.L., et al. Structure and Function Analysis of an Antibody Recognizing All Influenza A Subtypes. Cell 166, 596-608 (2016).

22. Wang, W., et al. Human antibody 3E1 targets the HA stem region of H1N1 and H5N6 influenza A viruses. Nat Commun 7, 13577 (2016).

23. Ekiert, D.C., et al. A highly conserved neutralizing epitope on group 2 influenza A viruses. Science 333, 843-850 (2011).

24. Yi, C., et al. Junctional and somatic hypermutation-induced CX4C motif is critical for the recognition of a highly conserved epitope on HCV E2 by a human broadly neutralizing antibody. Cell Mol Immuno/ 18, 675-685 (2021).

25. Law, M., et al. Broadly neutralizing antibodies protect against hepatitis $\mathrm{C}$ virus quasispecies challenge. Nat Med 14, 25-27 (2008).

26. Huang, J., et al. Broad and potent neutralization of HIV-1 by a gp41-specific human antibody. Nature 491, 406-412 (2012). 
27. Walker, L.M., et al. Broad neutralization coverage of HIV by multiple highly potent antibodies. Nature 477, 466-470 (2011).

28. Wang, C., et al. A conserved immunogenic and vulnerable site on the coronavirus spike protein delineated by cross-reactive monoclonal antibodies. Nat Commun 12, 1715 (2021).

29. Sauer, M.M., et al. Structural basis for broad coronavirus neutralization. Nat Struct Mol Bio/ 28, 478-486 (2021).

30. Zhou, P., et al. A protective broadly cross-reactive human antibody defines a conserved site of vulnerability on beta-coronavirus spikes. bioRxiv (2021).

31. Pinto, D., et al. Broad betacoronavirus neutralization by a stem helix-specific human antibody. Science(2021).

32. Tortorici, M.A., et al. Broad sarbecovirus neutralization by a human monoclonal antibody. Nature (2021).

33. Corti, D., Purcell, L.A., Snell, G. \& Veesler, D. Tackling COVID-19 with neutralizing monoclonal antibodies. Cel/ 184, 3086-3108 (2021).

34. Benton, D.J., et al. Receptor binding and priming of the spike protein of SARS-CoV-2 for membrane fusion. Nature 588, 327-330 (2020).

35. Cai, Y., et al. Distinct conformational states of SARS-CoV-2 spike protein. Science 369, 1586-1592 (2020).

36. Xia, S., et al. Inhibition of SARS-CoV-2 (previously 2019-nCoV) infection by a highly potent pancoronavirus fusion inhibitor targeting its spike protein that harbors a high capacity to mediate membrane fusion. Cell Res 30, 343-355 (2020).

37. Zhu, Y., Yu, D., Yan, H., Chong, H. \& He, Y. Design of Potent Membrane Fusion Inhibitors against SARS-CoV-2, an Emerging Coronavirus with High Fusogenic Activity. J Viro/ 94(2020).

38. Wrapp, D., et al. Cryo-EM structure of the 2019-nCoV spike in the prefusion conformation. Science $367,1260-1263$ (2020).

39. Hoffmann, M., et al. SARS-CoV-2 Cell Entry Depends on ACE2 and TMPRSS2 and Is Blocked by a Clinically Proven Protease Inhibitor. Cel/ 181, 271-280 e278 (2020).

40. Belouzard, S., Chu, V.C. \& Whittaker, G.R. Activation of the SARS coronavirus spike protein via sequential proteolytic cleavage at two distinct sites. Proc Natl Acad Sci U S A 106, 5871-5876 (2009).

41. Tang, T., Bidon, M., Jaimes, J.A., Whittaker, G.R. \& Daniel, S. Coronavirus membrane fusion mechanism offers a potential target for antiviral development. Antiviral Res 178, 104792 (2020). 
42. Walls, A.C., et al. Tectonic conformational changes of a coronavirus spike glycoprotein promote membrane fusion. Proc Natl Acad Sci U S A 114, 11157-11162 (2017).

43. Jiang, L., et al. Potent neutralization of MERS-CoV by human neutralizing monoclonal antibodies to the viral spike glycoprotein. Sci Trans/ Med 6, 234 ra259 (2014).

44. Giang, E., et al. Human broadly neutralizing antibodies to the envelope glycoprotein complex of hepatitis C virus. Proc Natl Acad Sci U S A 109, 6205-6210 (2012).

45. Poh, C.M., et al. Two linear epitopes on the SARS-CoV-2 spike protein that elicit neutralising antibodies in COVID-19 patients. Nat Commun 11, 2806 (2020).

46. Ren, H. \& Zhou, P. Epitope-focused vaccine design against influenza A and B viruses. Curr Opin Immunol 42, 83-90 (2016).

47. Correia, B.E., et al. Proof of principle for epitope-focused vaccine design. Nature 507, 201-206 (2014).

48. Yi, C., et al. Key residues of the receptor binding motif in the spike protein of SARS-CoV-2 that interact with ACE2 and neutralizing antibodies. Cell Mol Immunol 17, 621-630 (2020).

49. Yuan, Y., et al. Cryo-EM structures of MERS-CoV and SARS-CoV spike glycoproteins reveal the dynamic receptor binding domains. Nat Commun 8, 15092 (2017).

50. Walls, A.C., et al. Glycan shield and epitope masking of a coronavirus spike protein observed by cryo-electron microscopy. Nat Struct Mol Biol 23, 899-905 (2016).

51. Tortorici, M.A., et al. Structural basis for human coronavirus attachment to sialic acid receptors. Nat Struct Mol Biol 26, 481-489 (2019).

52. Li, Z., et al. The human coronavirus HCoV-229E S-protein structure and receptor binding. Elife 8(2019).

53. Chan, K.K., et al. Engineering human ACE2 to optimize binding to the spike protein of SARS coronavirus 2. Science 369, 1261-1265 (2020).

\section{Figures}



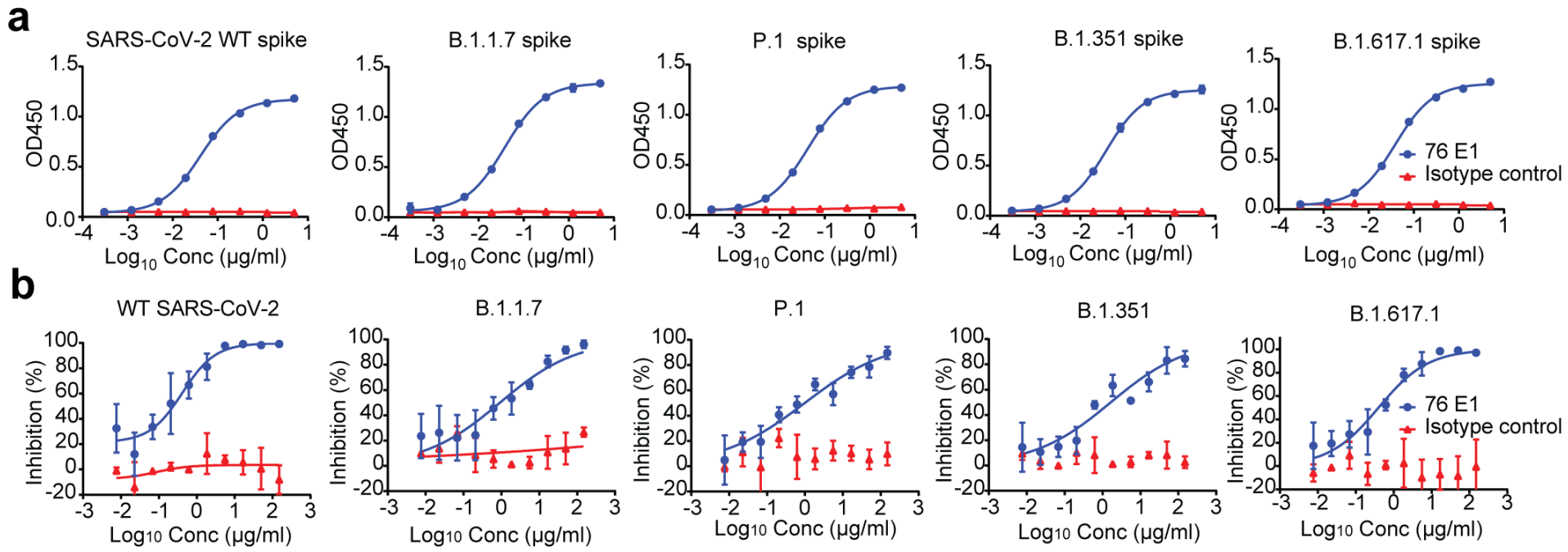

C

\begin{tabular}{|c|c|c|c|c|}
\hline & \multicolumn{2}{|c|}{$76 \mathrm{E} 1$} & \multicolumn{2}{|c|}{ Isotype control } \\
\hline & EC50 $(\mu \mathrm{g} / \mathrm{ml})$ & IC50 ( $\mu \mathrm{g} / \mathrm{ml})$ & EC50 $(\mu \mathrm{g} / \mathrm{ml})$ & $\mathrm{IC50}(\mu \mathrm{g} / \mathrm{ml})$ \\
\hline WT SARS-CoV-2 & 0.041 & 0.418 & $>5$ & $>150$ \\
\hline B.1.1.7 & 0.038 & 0.913 & $>5$ & $>150$ \\
\hline P.1 & 0.042 & 0.984 & $>5$ & $>150$ \\
\hline B.1.351 & 0.038 & 1.863 & $>5$ & $>150$ \\
\hline B.1.617.1 & 0.039 & 0.397 & $>5$ & $>150$ \\
\hline
\end{tabular}
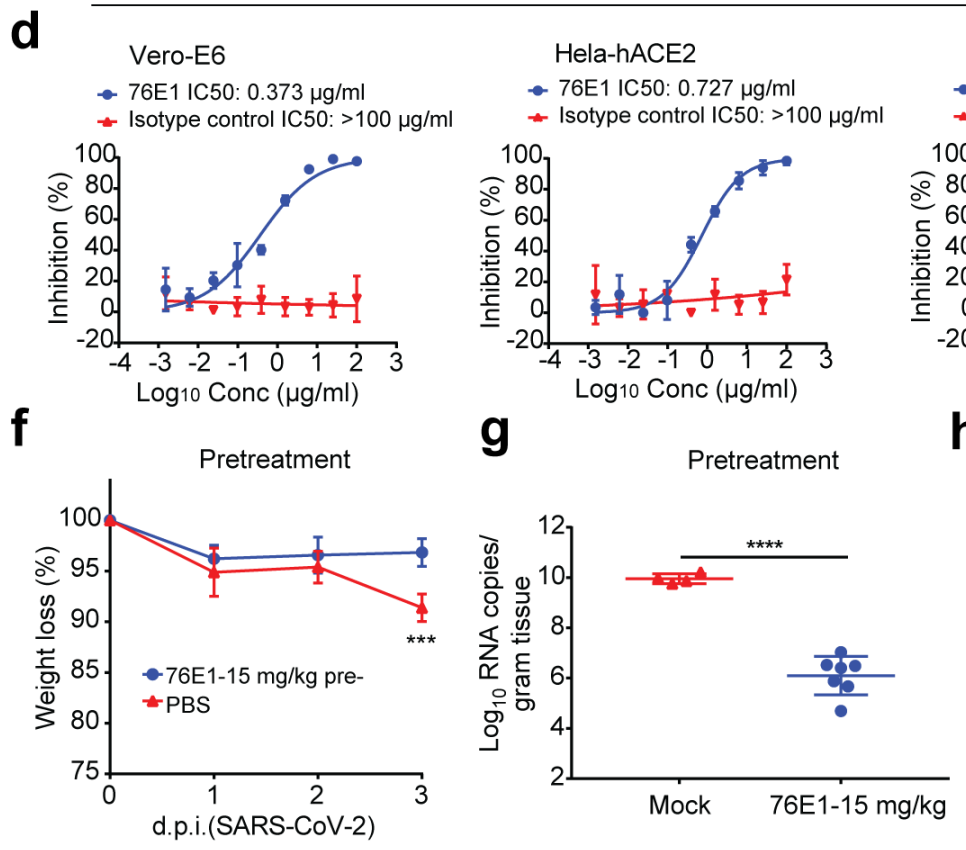

Calu-3

- 76E1 IC50: $0.433 \mu \mathrm{g} / \mathrm{ml}$

- Isotype control IC50: >100 $\mu \mathrm{g} / \mathrm{ml}$

e

Mice study outline:

Pre-treatment:

76E1 or PBS Infection:

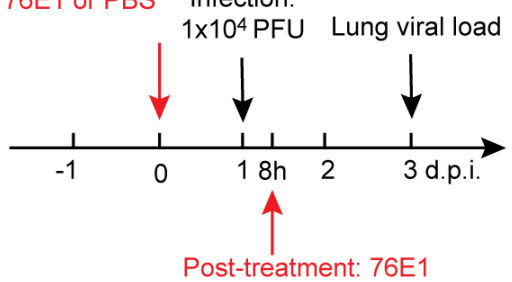

Pretreatment: 76E1 $(15 \mathrm{mg} / \mathrm{kg})$

Posttreatment: 76E1 (25 mg/kg)

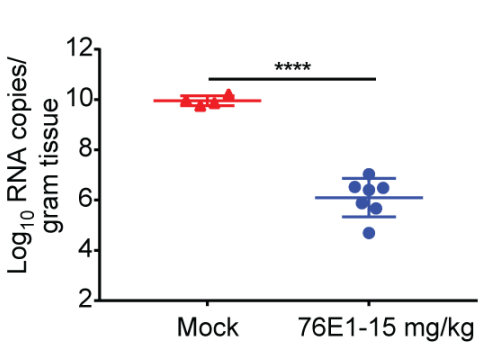

h

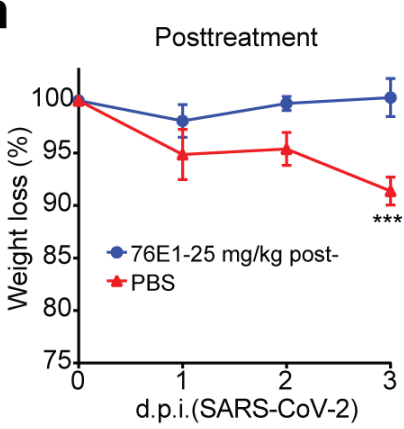

i

Posttreatment

\section{Figure 1}

Neutralization of SARS-CoV-2 by 76E1 in vitro and in vivo. a. The dose-dependent binding curves of 76E1 to the soluble S-ECD proteins of SARS-CoV-2 and its variants of concern, including B.1.1.7, P.1, B.1.351 and B.1.617.1. b. 76E1-mediated neutralization of pseudotyped SARS-CoV-2 and its variants in 293TACE2 cells. c. The summary of the binding EC50s and neutralization IC50s of 76E1 against SARS-CoV-2 and its variants. $d$. Authentic SARS-CoV-2 neutralization of 76E1 performed using Vero-E6, HeLa-hACE2 or Calu-3 target cells. The curves were fit by nonlinear regression and the data are expressed as the mean \pm SD of at least two replicate wells. Representative data are shown from at least two independent experiments. e-i. Protective efficacy of 76E1 against authentic SARS-CoV-2 infection in hACE2 transgenic 
mice. e. The study outline. Pretreatment: the mice were infused with $15 \mathrm{mg} / \mathrm{kg} 76 \mathrm{E} 1$ or PBS intraperitoneally one day before inoculating the mice with 1x104 PFU SARS-CoV-2 via intranasal infection. Posttreatment: the mice were treated with $25 \mathrm{mg} / \mathrm{kg} 76 \mathrm{E} 18 \mathrm{~h}$ after SARS-CoV-2 infection. The pre- or posttreatment experiments were concurrently conducted and the PBS administrated mice were included as control mice for both experiments. The mice body weight from pretreatment (f) and posttreatment $(\mathrm{h})$ groups were monitored for 3 days after infection. Lung virus titres of each mouse from pretreatment (g) and posttreatment (i) groups at 3 d.p.i. were determined by qRT-PCR with two replicates. Results are depicted as the mean \pm SD. The $P$ values are analysed using the Multiple $t$ tests. ${ }^{*} * *<0.001$, $\star * * * P<0.0001$.

a

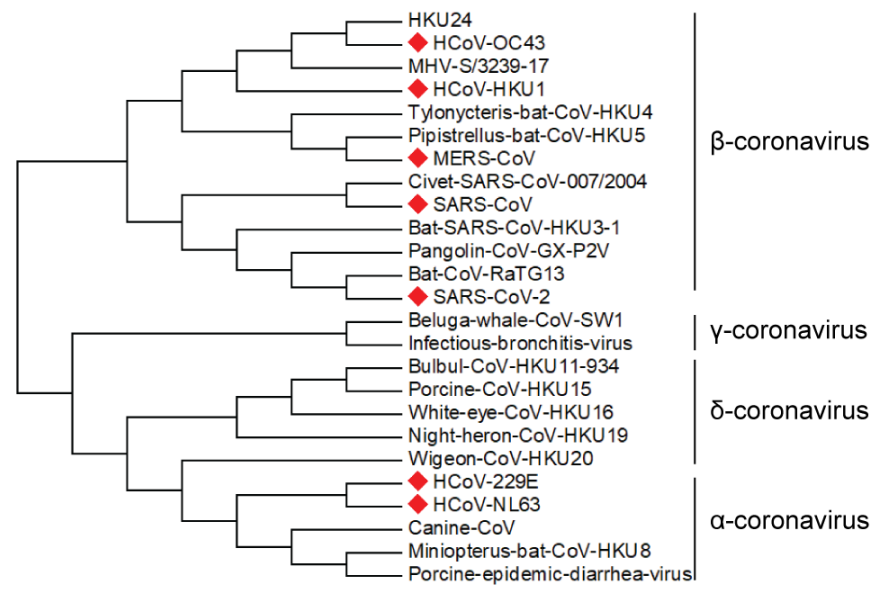

b

\begin{tabular}{|c|c|c|c|c|c|c|}
\hline $\begin{array}{l}\text { Human } \\
\text { viruses }\end{array}$ & $\begin{array}{c}\text { EC50 } \\
(\mu \mathrm{g} / \mathrm{ml})\end{array}$ & $\begin{array}{l}\mathrm{KD} \\
(\mathrm{M})\end{array}$ & $\begin{array}{c}\text { Kon } \\
\text { (1/Ms) }\end{array}$ & $\begin{array}{l}\text { Koff } \\
(1 / s)\end{array}$ & $\begin{array}{l}\text { Pseudovirus } \\
\text { IC50 }(\mu \mathrm{g} / \mathrm{ml})\end{array}$ & $\begin{array}{l}\text { Authentic virus } \\
\text { IC50 }(\mu \mathrm{g} / \mathrm{ml})\end{array}$ \\
\hline HCoV-229E & 0.110 & 1.29E-09 & $3.85 E+04$ & 4.96E-05 & 1.164 & 1.595 \\
\hline HCoV-NL63 & 0.073 & 1.69E-09 & 4.30E+04 & 7.24E-05 & n.d. & 3.943 \\
\hline HCoV-OC43 & 0.042 & $<1.0 \mathrm{E}-12$ & $5.76 \mathrm{E}+04$ & $<1.0 \mathrm{E}-07$ & n.d. & 4.655 \\
\hline HCoV-HKU1 & 0.077 & $9.03 \mathrm{E}-11$ & $2.72 E+04$ & 2.45E-06 & n.d. & n.d. \\
\hline SARS-CoV & 0.085 & $1.07 \mathrm{E}-10$ & $4.31 E+04$ & 4.61E-06 & 4.821 & n.d. \\
\hline MERS-CoV & 0.038 & 1.03E-09 & $8.15 E+04$ & 8.38E-05 & 3.825 & n.d. \\
\hline SARS-CoV-2 & 0.072 & 4.31E-10 & $5.16 E+04$ & 2.23E-05 & 0.418 & 0.373 \\
\hline
\end{tabular}

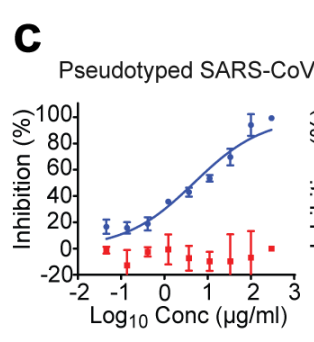

i
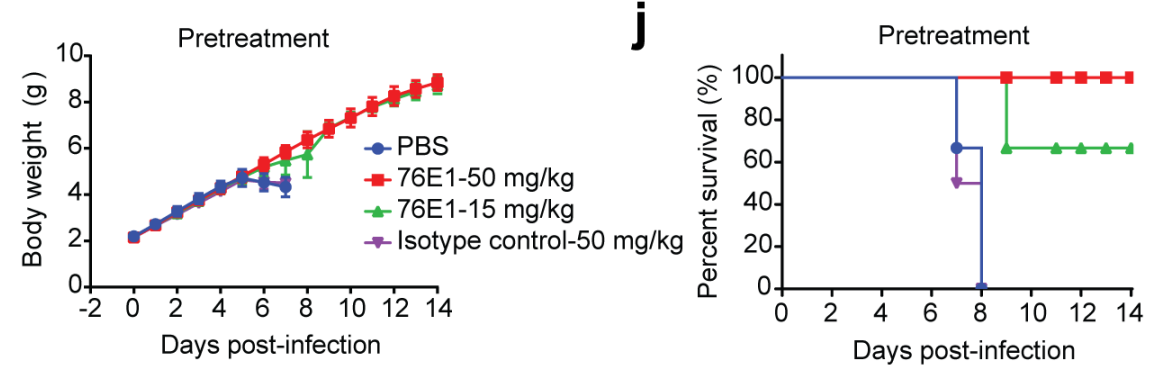

m
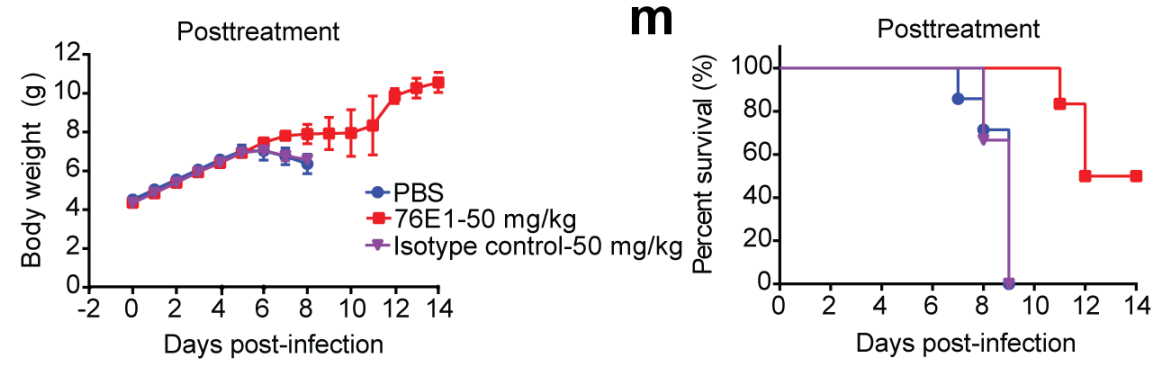

n

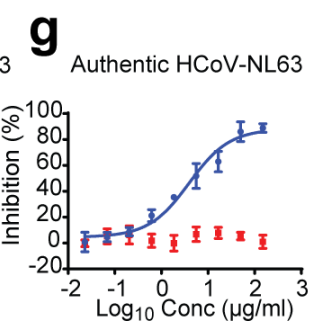

h Authentic HCoV-229E
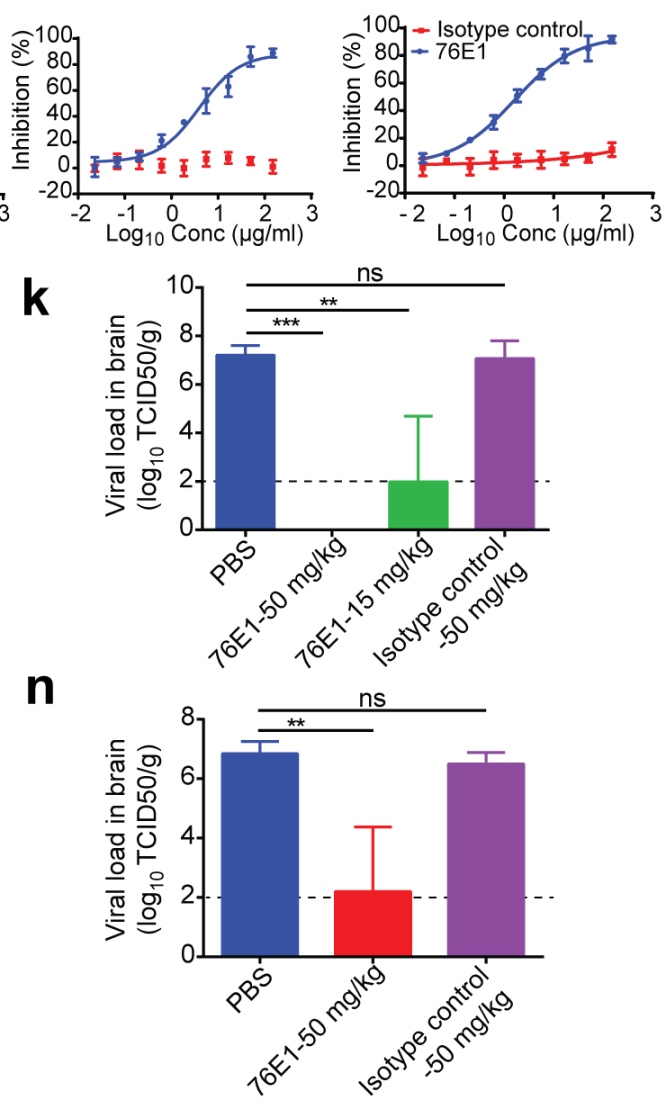
Broad binding and neutralization of 76E1 to multiple $\alpha$ - and $\beta$-coronaviruses. a. Phylogenetic tree of 28 coronaviruses constructed via MEGA (Molecular Evolutionary Genetics Analysis) and maximum likelihood analysis of full spike amino acid sequences downloaded from the NCBI (National Center for Biotechnology Information) database. Seven human coronaviruses were denoted in filled red diamond. b. Binding affinity of $76 \mathrm{E} 1$ to soluble S-ECD proteins of the seven human coronaviruses measured by BLI and ELISA. n.d., not determined. c-e. 76E1-mediated neutralization of pseudotyped SARS-CoV in 293ThACE2 cells and MERS-CoV and HCoV-229E in HuH-7 cells. f-h, 76E1-mediated neutralization of authentic HCoV-OC43 in RD cells, HCoV-NL63 in LLC-MK2 cells and HCoV-229E in HuH-7 cells. i-k, Prophylactic efficacy of 76E1 in mice against OC43. Newborn mice were pretreated with 76E1 or isotype control antibody (15 or $50 \mathrm{mg} / \mathrm{kg}$ ) or PBS 24h before challenge with a lethal dose of OC43. Body weight (i) and survival rate (j) were monitored for 14 days after infection. $k$, Virus titres in brain of each mouse was determined by calculating CPE on RD cells and expressed as TCID50/g. I-n, Protective efficacy of 76E1 treatment after $\mathrm{OC} 43$ infection in mice. Newborn mice were treated with 76E1 or isotype control antibody $(50 \mathrm{mg} / \mathrm{kg}$ ) or PBS $2 \mathrm{~h}$ after challenge with OC43. Body weight $(\mathrm{l})$ and survival rate $(\mathrm{m})$ were monitored for 14 days after infection. $n$, Virus titres in brain of each mouse. Representative data are shown from at least two independent experiments. Results are depicted as the mean \pm SD. The $P$ values are analysed using the Multiple t tests. ${ }^{\star *} \mathrm{P}<0.01,{ }^{* \star *} \mathrm{P}<0.001, \mathrm{~ns}$, not significant. 
a

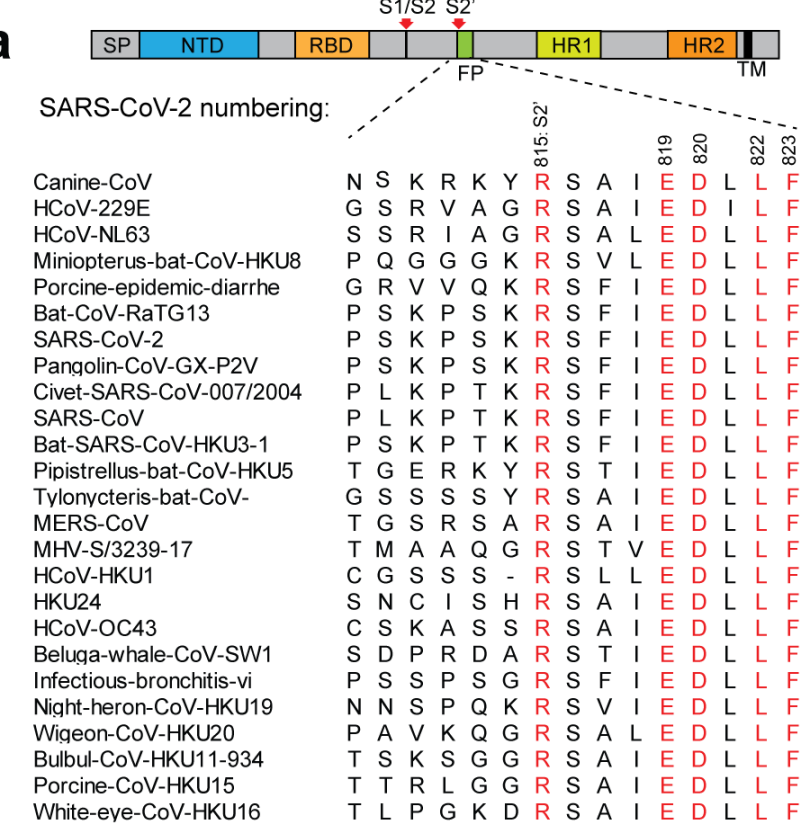

C Mutants Sequence $\quad 76 \mathrm{E} 1 \quad$ Isotype control EC50 Fold change EC50 Fold change

WT PSKPSKRSFIEDLLE $0.043 \quad 1.00 \quad$ n.b. n.b.

P809A ASKPSKRSFIEDLLE $0.044 \quad 1.00 \quad$ n.b. n.b.

S810A PAKPSKRSFIEDLLE $0.043 \quad 1.00 \quad$ n.b. n.b.

K811A PSAPSKRSFIEDLLE $0.043 \quad 1.00 \quad$ n.b. n.b.

P812A PSKASKRSEIEDLLE $0.044 \quad 1.01 \quad$ n.b. n.b.

S813A PSKPAKRSFIEDLLE $0.066 \quad 1.53 \quad$ n.b. n.b.

K814A PSKPSARSFIEDLLE $0.050 \quad 1.16 \quad$ n.b. n.b.

R815A PSKPSKASFIEDLLE n.b. n.b. n.b. n.b.

S816A PSKPSKRAFIEDLLE $0.052 \quad 1.20 \quad$ n.b. n.b.

F817A PSKPSKRSAIEDLLF $0.058 \quad 1.33 \quad$ n.b. n.b.

I818A PSKPSKRSFAEDLLE $0.056 \quad 1.30 \quad$ n.b. n.b.

E819A PSKPSKRSEIADLLE n.b. n.b. n.b. n.b.

D820A PSKPSKRSEIEALLE $1.045 \quad 24.13$ n.b. n.b.

L821A PSKPSKRSFIEDALE $0.043 \quad 0.99 \quad$ n.b. n.b.

L822A PSKPSKRSFIEDLAF $2.220 \quad 51.27 \quad$ n.b. n.b.

F823A PSKPSKRSFIEDLLA n.b. n.b. n.b. n.b.

9 a-coronavirus peptide

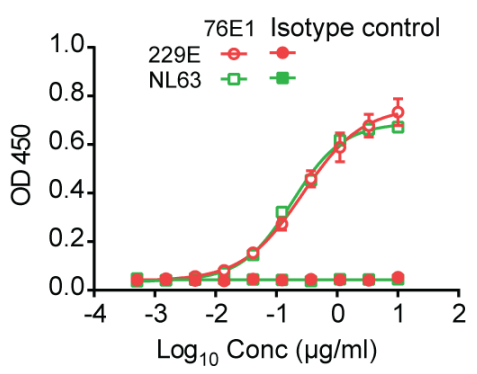

h $\beta$-coronavirus peptide

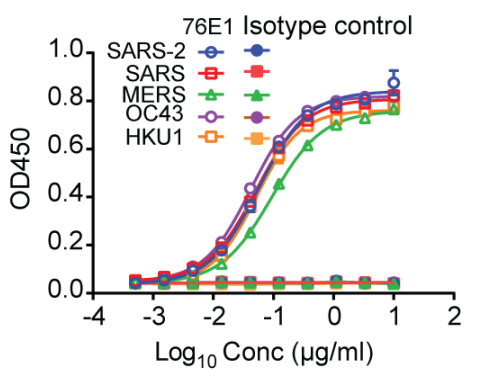

b
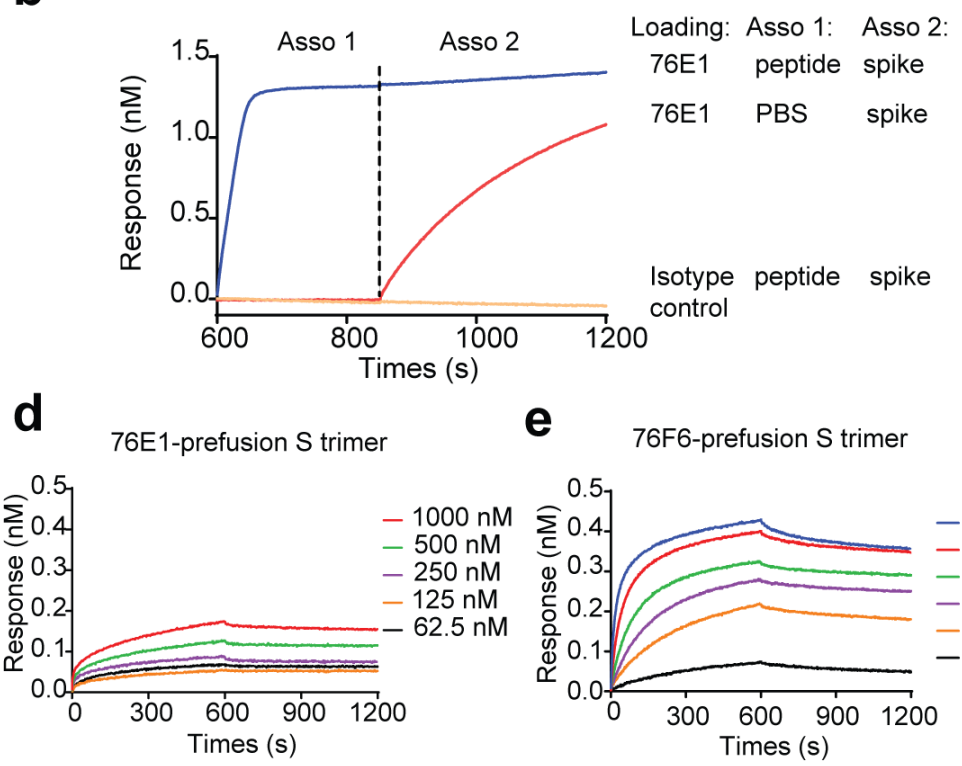

e 76F6-prefusion S trimer
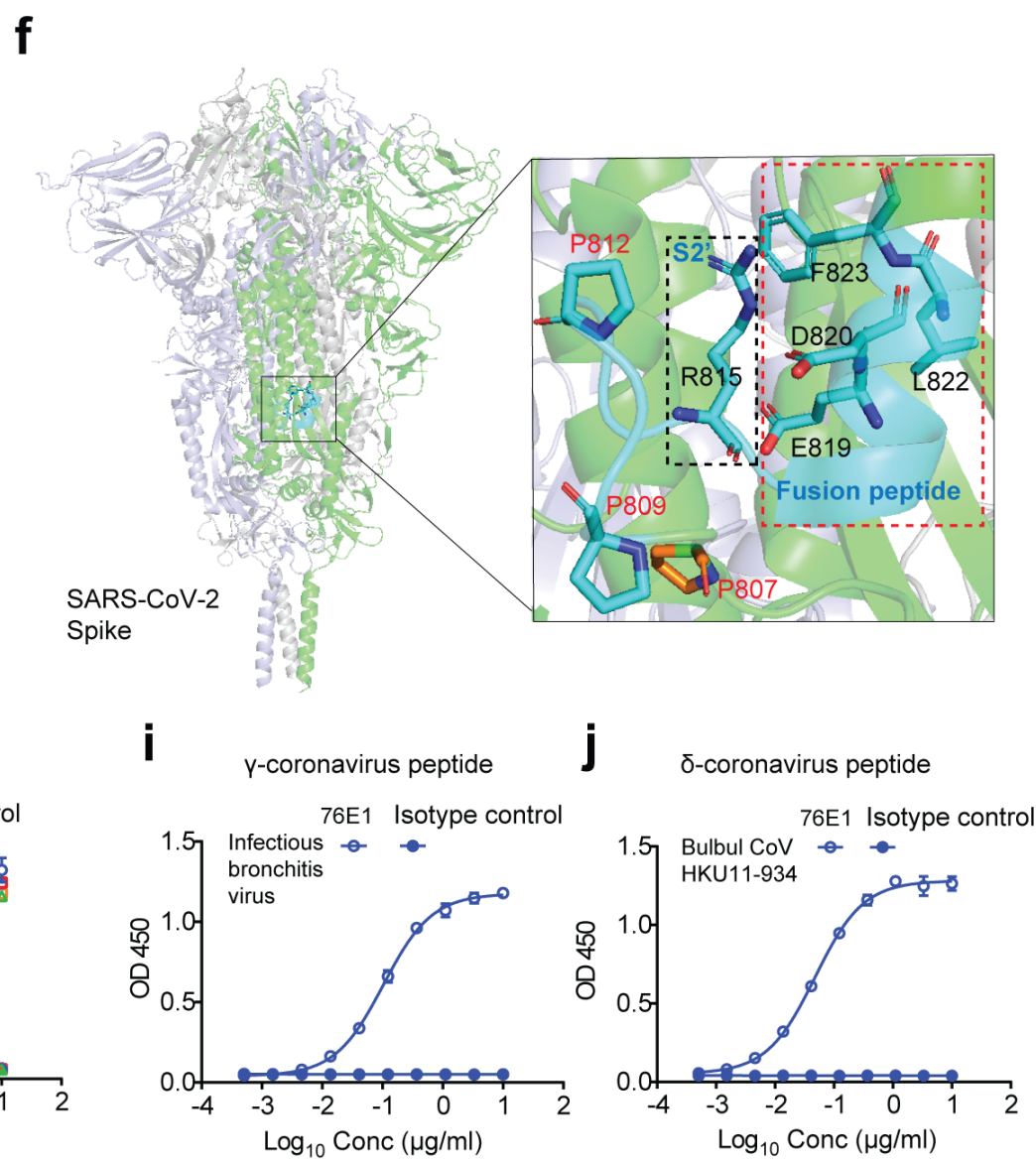

\section{Figure 3}

Antibody 76E1 targets a highly conserved epitope within S2' cleavage site and fusion peptide. a. Schematic representation of spike protein. SP, signal peptide; NTD, N-terminal domain; RBD, receptor binding domain; FP, fusion peptide; HR1 and HR2, heptad repeat domain; TM, transmembrane domain; S1/S2 and S2' indicate the cleavage sites. 28 coronavirus peptide809-823 (SARS-CoV-2 numbering) from $a, \beta, \gamma$ and $\delta$ genera was aligned. b. Epitope competition of the peptide and SARS-CoV-2 S protein with 
$76 \mathrm{E} 1$ measured by BLI. Asso, association. c, ELISA-based epitope alanine scanning on the peptide809823 of SARS-CoV-2, shown as EC50 ( $\mu \mathrm{g} / \mathrm{ml})$ and EC50 fold change compared with WT peptide. The key epitope residues are annotated in Figure 3A shown in red colour. d-e. The binding affinity of 76E1 and 76F6 (an RBD antibody isolated in this project) to the prefusion trimeric SARS-CoV-2 spike protein was determined by BLI. f. Cartoon display of spike809-823 on the overall structure of the prefusion trimeric spike protein of SARS-CoV-2 from the side view (PDB ID: 6XR8). One protomer is highlighted in green, and the other two copies in the trimer are coloured light grey and light purple. Peptide809-823 is coloured light blue. Functionally defined epitope representation of 76E1 with the side chains shown and annotated in black on the enlarged view of SARS-CoV-2 spike809-823. The three amino acids proline preceding S2' cleavage site are specifically marked in red. g-j. 76E1 broadly reacts with spike peptides809-823 of all four coronavirus genera. ELISA-based binding activity of 76E1 to the spike peptides809-823 of coronavirus from $a(g), \beta(h), Y(i)$ and $\delta(j)$ genera. Representative data are shown from at least two independent experiments. Results are depicted as the mean \pm SD.

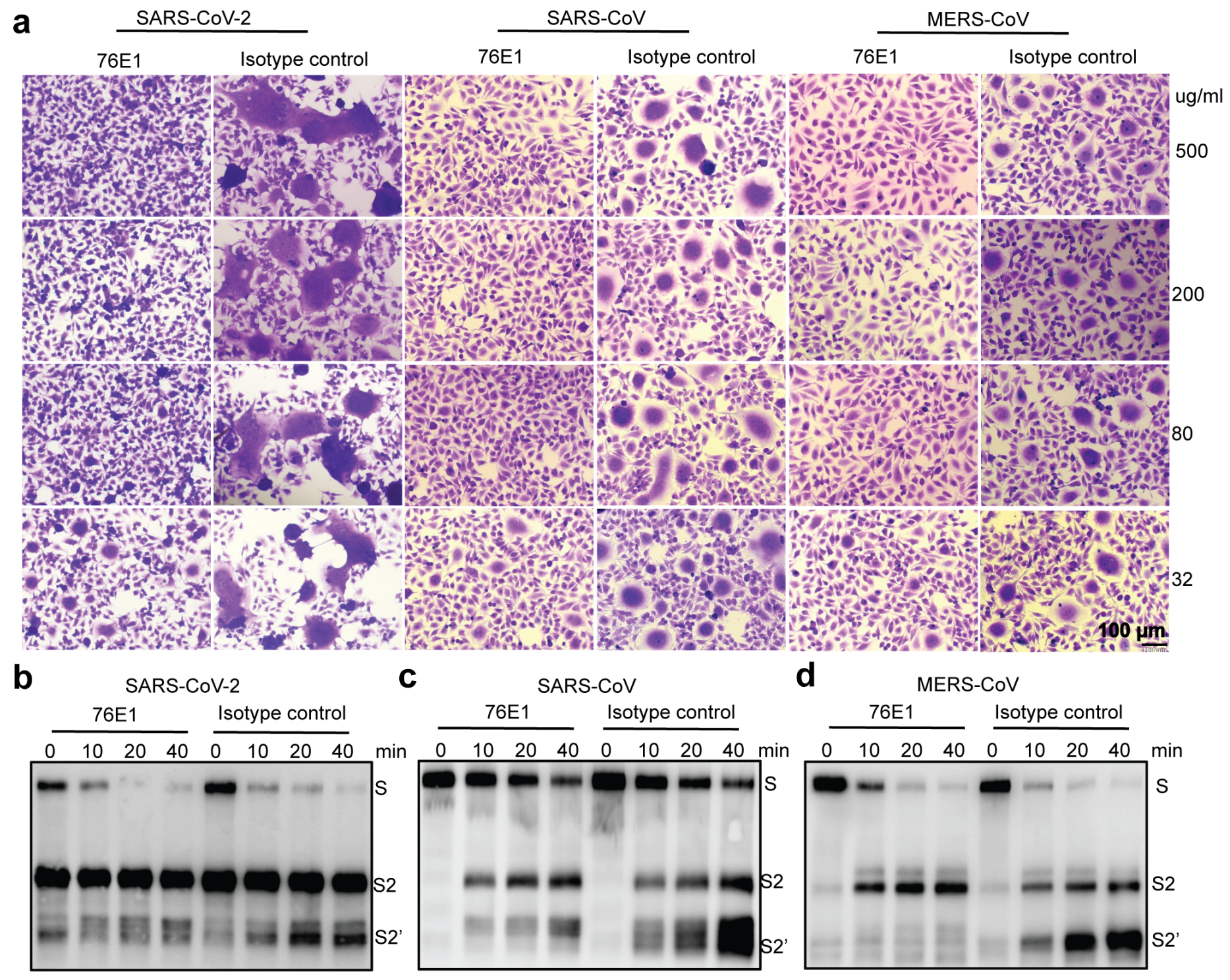

Figure 4 
76E1 inhibits membrane fusion by inhibiting S2' cleavage. a. 76E1 inhibits spike- and ACE2-mediated cellcell membrane fusion. 76E1 was serially diluted and incubated with HeLa cells transfected with the $S$ proteins of SARS-CoV-2, SARS-CoV or MERS-CoV, which were then mixed with HeLa cells transfected with ACE2. The scale bar is shown as $100 \mu \mathrm{m}$. b-d. 76E1 inhibits trypsin-induced S2' cleavage. Addition of $76 E 1$ to the C-terminal tagged S-ECD protein of SARS-CoV-2 (b), SARS-CoV (c) or MERS-CoV (d) before treatment with trypsin inhibits the cleavage of the spike proteins in a time-dependent manner, but addition of the isotype control antibody does not. An anti-HIS-HRP antibody was used for the detection. S2, approximately 70 KD, S2', approximately 55KD. Representative data are shown from at least two independent experiments.

\section{a}

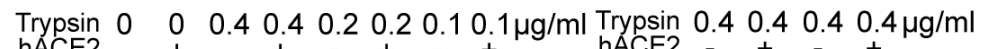
hACE2

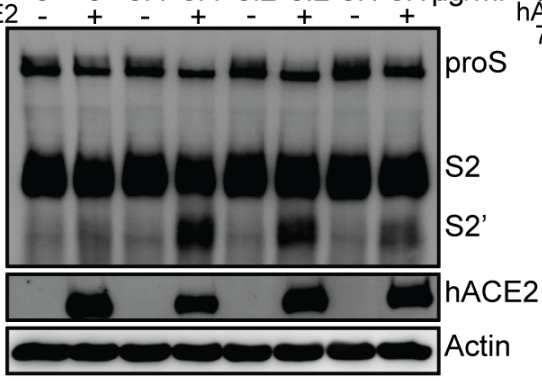

d

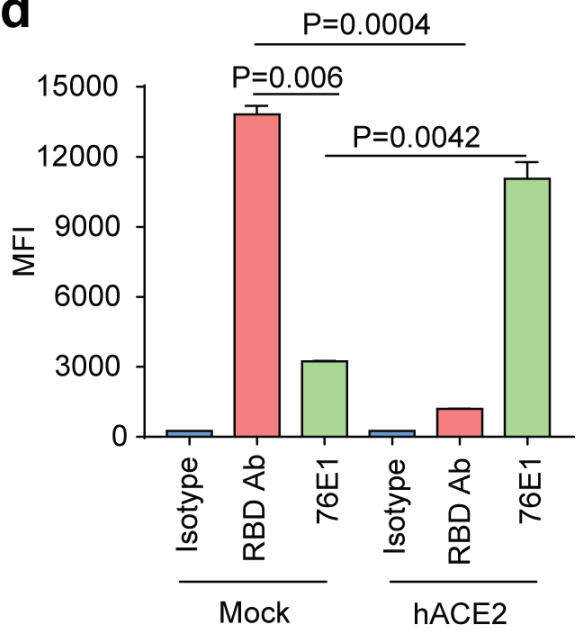

b

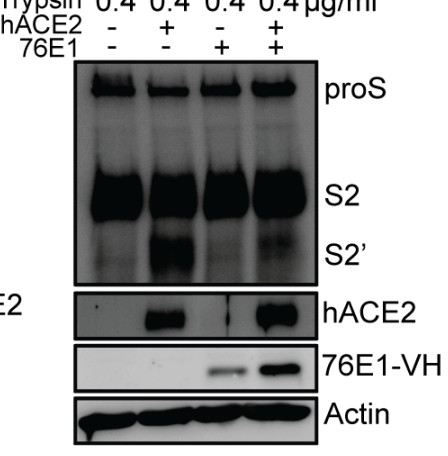

e

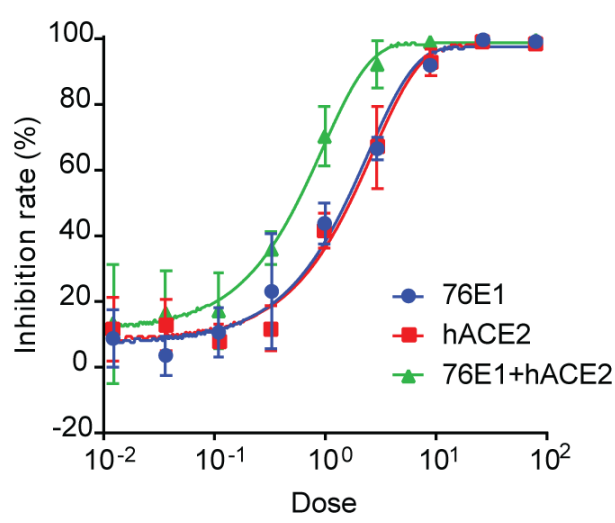

C
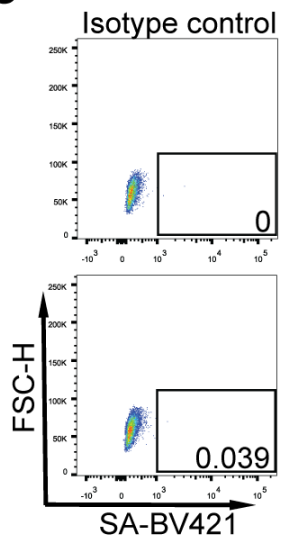
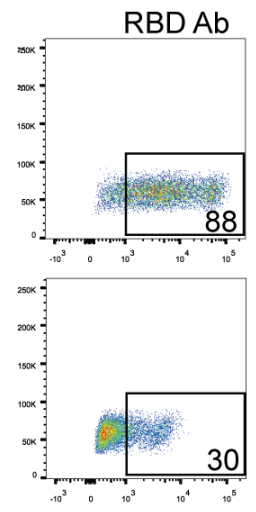

f
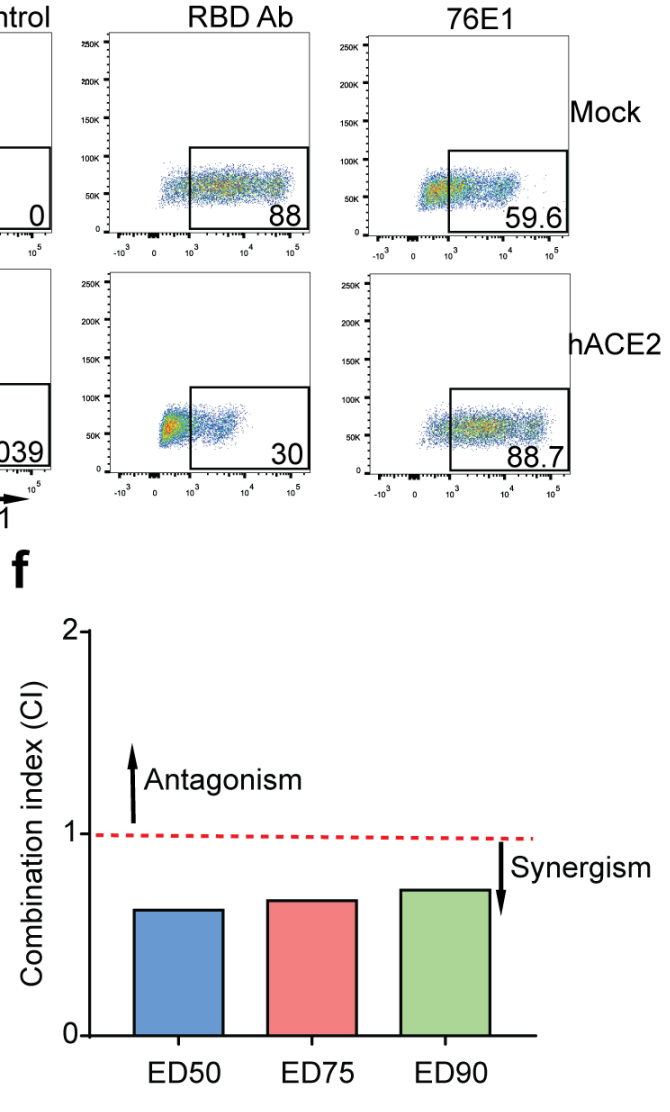

g

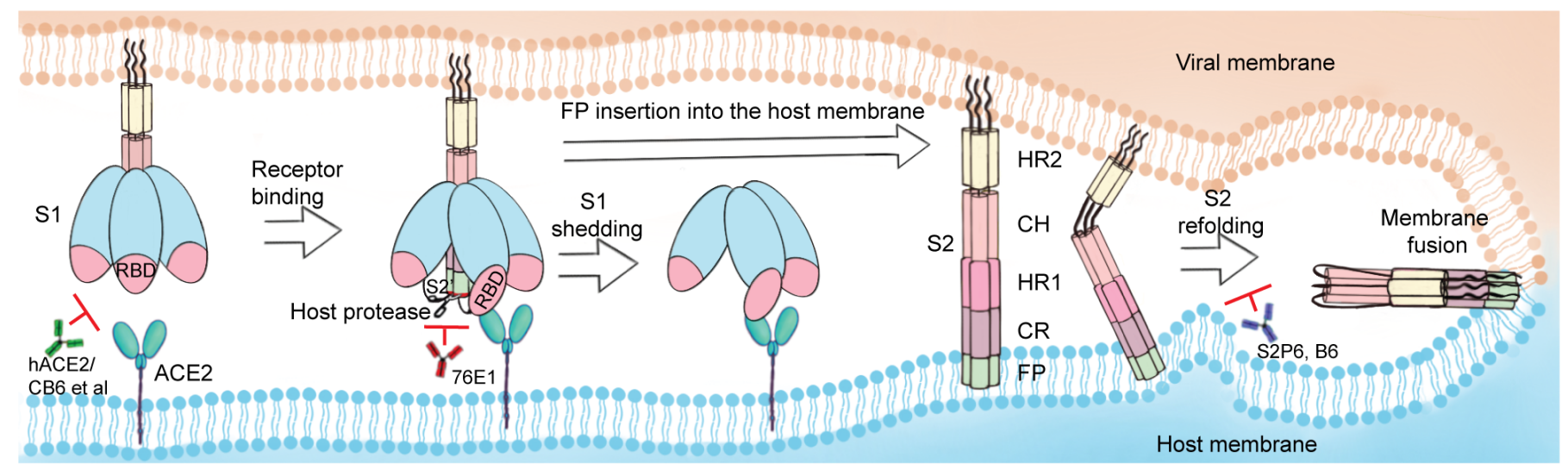


Receptor binding to ACE2 facilitates the function of 76E1. a, hACE2 promoted trypsin-induced S2' cleavage. A549 cells stably expressing SARS-CoV-2 spike protein (A549-S cells) were incubated with hACE2 for $45 \mathrm{~min}$ before treatment with $0.4,0.2$ or $0.1 \mu \mathrm{g} / \mathrm{ml}$ trypsin for $15 \mathrm{~min}$. An anti-S2 rabbit antibody was used to detect the pro-S, S2 and S2' fragments. Recombinant hACE2 tagged with human Fc was expressed and detected using an anti-human Fc antibody. b, 76E1 inhibited hACE2 and trypsininduced S2' cleavage. A549-S cells were incubated with an hACE2 and 76E1 mixture for 45 min before trypsin treatment at $0.4 \mu \mathrm{g} / \mathrm{ml}$ for $15 \mathrm{~min}$. S2' cleavage decreased in the presence of 76E1. c-d, hACE2 facilitated 76E1 binding to the cell surface-expressed spike proteins of SARS-CoV-2, as determined by flow cytometry. The original cell frequencies (c) and the average fluorescence intensity (MFI) values (d) are shown. e-f, The synergistic effect of 76E1 and hACE2 in neutralizing authentic SARS-CoV-2. e, Neutralizing curves of 76E1 and hACE2 alone and in combination at a constant ratio with serial threefold diluted concentration from 81 times IC50. A dose of 1 represents the concentration at IC50. The constant ratio indicates the ratio between 76E1 and hACE2 at IC50. $\mathrm{f}, \mathrm{Cl}$ (combination index) values at ED50, ED75 and ED90 (50\%, 75\% and 90\% effective dose). The $\mathrm{Cl}$ values was calculated using the CompuSyn program. The dotted line in red represents $\mathrm{Cl}=1$ below of which was defined as synergism versus top of which as antagonism. Representative data are shown from at least two independent experiments. Results are depicted as the mean $\pm S D$. The $P$ values were analysed using the unpaired $t$ test. ${ }^{*} P<0.05$, ${ }^{*} P<$ $0.01,{ }^{\star \star \star} P<0.001,{ }^{\star \star \star \star} P<0.0001$. g, Proposed model for mAbs and soluble hACE2 to neutralize SARSCoV-2. For the closed RBD in the prefusion $S$ trimer, it could not recognize the hACE2 receptor of the host cell while occasionally, one RBD may hold the up conformation through rearrangement and bind to hACE2. The receptor binding process releases the structural constraints on the fusion peptide and S2' cleavage site and induced obvious conformational change of spike protein, especially for the exposure of the fusion peptide (FP) and S2' site. Once the FP and S2' site exposed, 76E1 will immediately target the sites and inhibit the S2' cleavage, thus avoid a cascade of conformational rearrangement and block the virus-host cell membrane fusion. Most of the RBD antibodies, such as CB6 and the soluble hACE2 protein competitively bind to the virus spike protein and block virus recognizing cell surface-expressed receptor ACE2. Another class of antibodies targeting the peptide 1147-SFKEELDKYFKNKTS-1161 of S2 region, such as S2P6 and B6 were reported to disrupt the stem helix bundle, prevent S2 refolding from the pre- to the postfusion state and thus block virus entry though inhibiting membrane fusion. Therefore, 76E1 represent a new class of antibody.

\section{Supplementary Files}

This is a list of supplementary files associated with this preprint. Click to download.

- 76E1ExtendedData20210923.docx

- 76E1Supplementaryinformation20210923.docx 